\title{
HIGH-PRECISION DECADAL CALIBRATION OF THE RADIOCARBON TIME SCALE, AD 1950-6000 BC
}

\section{MINZE STUIVER}

Department of Geological Sciences and Quaternary Research Center, University of Washington Seattle, Washington 98195 USA

and

\section{BERND BECKER}

Institute für Botanik, Universität Hohenheim, D-7000 Stuttgart 70 Germany

\section{INTRODUCTION}

The radiocarbon ages of dendrochronologically-dated wood samples, each covering 10 years, are now available for the cal AD 1950-6000 BC age range. The decadal calibration curve constructed from these data comprises 1) the previously published AD 1950-2500 BC portion (Stuiver \& Becker 1986), to which minor ${ }^{14} \mathrm{C}$ age corrections were applied, and 2) the new 2500-6000 BC extension.

The calibration error (standard deviation in ${ }^{14} \mathrm{C}$ age) is based on 1) an estimate of the reproducibility in the ${ }^{14} \mathrm{C}$ age determination in the Seattle laboratory, and 2) interlaboratory comparisons that provide information on the sum total of uncertainty tied to the processes of wood allocation, dendro-age determination, sample pretreatment, laboratory ${ }^{14} \mathrm{C}$ determination, regional ${ }^{14} \mathrm{C}$ distribution and ${ }^{14} \mathrm{C}$ differences between individual trees of the same chronology (Stuiver \& Pearson 1992, 1993). The standard deviations of the Table $1{ }^{14} \mathrm{C}$ calibration data for the $\mathrm{AD}$ 1950-2500 and 2500-6000 BC intervals are, respectively, equal to 1.6 and 1.7 times the standard deviation derived from the near-Gaussian counting statistics of the accumulated number of counts for the sample and standards.

The ${ }^{14} \mathrm{C}$ ages for the decadal wood samples (Table 1) were used to construct the bidecadal Seattle-Belfast calibration curve reported in this issue (Stuiver \& Pearson 1993; Pearson \& Stuiver 1993). The ${ }^{14} \mathrm{C}$ age errors are substantially smaller for the bidecadal curve, as most bidecadal data points were obtained by averaging 2 Seattle decadal ${ }^{14} \mathrm{C}$ ages, and 1 Belfast bidecadal ${ }^{14} \mathrm{C}$ age. Thus, the bidecadal curves should be used for most purposes. However, the decadal curve is more important when accounting for the fine structure of ${ }^{14} \mathrm{C}$ age calibration of samples formed during intervals of a decade or less.

\section{DENDROCHRONOLOGY AND SAMPLE TREATMENT}

The trees used for the AD interval were mainly from the US Pacific Northwest or California (Table 2 in Stuiver \& Becker 1986). Most of the BC material was dendrochronologically dated wood from the German Main-Donau chronology (Becker 1993). Thirteen samples from the US bristlecone pine chronology (Ferguson \& Graybill 1983) were ${ }^{14} \mathrm{C}$ dated as well. A limited number of samples from the Irish oak chronology (Pilcher et al. 1984) was used near 500 BC. Refer to Stuiver and Becker (1986) for wood pretreatment procedures. Cellulose, isolated from the decadal wood samples, was used for all $2500-6000 \mathrm{BC}{ }^{14} \mathrm{C}$ determinations. 


\section{CORRECTIONS}

At the start of our time-scale calibration efforts in 1973, the $\mathrm{CO}_{2}$ samples were stored in pyrex reservoirs for at least four weeks (Procedure 1). Radon decay took place during storage, and as a result, no detectable $\alpha$ particles were present in our $\alpha$ channel. Having never detected tree-ring radon in the $\alpha$ channels, we terminated their use some years later. Unfortunately, laboratory procedures were changed in 1976. Since then, tree-ring samples, after storage for four weeks, were purified on a self-circulating $\mathrm{Cu}$ oven (a pyrex reservoir filled with electrolytic copper wire and silver ribbon) at $450{ }^{\circ} \mathrm{C}$ before being admitted into the counter (Procedure 2). Small amounts of radon, most likely released from the walls of the heated reservoir, became part of the $\mathrm{CO}_{2}$ sample. Once the problem was recognized, we determined the count-rate differences (routinely four days of counting) of samples subjected either to Procedure 1 or Procedure 2 for three of our counters to be $\Delta \mathrm{Ra}=0.274 \pm 0.090,0.276 \pm 0.028$ and $0.319 \pm 0.090$ counts per minute (cpm) (weighted average, $0.279 \pm 0.026 \mathrm{cpm}$ ). Our radon correction for tree-ring samples (those measured for the 1977-1987 interval) is based on the above incremental four-day sample count-rate increase. As we kept Procedure 1 for routine (as opposed to high-precision) ${ }^{14} \mathrm{C}$ determinations, a radon correction is not needed for these samples.

The radon contribution can also be estimated from a comparison of the first- and last-day countrate differences of 1$)$ samples $(\Delta \mathrm{Sa})$, and 2$)$ oxalic acid and background standards $(\Delta \mathrm{Ox}$ and $\Delta \mathrm{B})$. Routine sample counts lasted four days; standards were routinely counted for three days. Procedure 2 was followed for all samples during the 1977-1987 period, and Procedure 1 for the standards, except for one counter where the oxalic acid $\mathrm{CO}_{2}$, after repurification, was subjected to Procedure 2. In this case, the radon contribution was also properly accounted for, but for simplicity, we discuss only the results obtained for Procedure 1 standard counts.

Each counter completes about 50 sample runs per year, as well as 25 runs of oxalic acid and background. Figure 1 gives the yearly averaged $\Delta \mathrm{Sa}$ and $\Delta \mathrm{B}$ values of the counter longest in operation (LC-4), together with $\Delta \mathrm{Ox}$ values of the LC-5 counter (for LC-4, the oxalic acid treatments did not always follow Procedure 1).

Procedure 1 background runs for $1977-1987$ yield $\Delta \mathrm{B}=0.025 \pm 0.017 \mathrm{cpm}$; Procedure 2 sample runs over the same interval yield $\Delta \mathrm{Sa}=0.189 \pm 0.049 \mathrm{cpm}$. As expected, lower $\Delta \mathrm{Sa}$ values $(0.035$ \pm 0.024 and $0.073 \pm 0.081 \mathrm{cpm}$ ) are encountered for the 1973-1975 and 1989-1990 periods, when only Procedure 1 was used. Further, oxalic acid runs with Procedure 1 only in counter LC-5 yield a 14-yr $\Delta \mathrm{Ox}$ average of $0.012 \pm 0.070 \mathrm{cpm}$ (Fig. 1).

First- and last-day sample count-rate differences, $\Delta \mathrm{Sa}$, were converted to $\Delta \mathrm{Ra}$, using the half-life of radon of slightly less than four days. For our tree-ring counters, we determined $\Delta \mathrm{Ra}$ values of $0.285 \pm 0.028,0.247 \pm 0.029$ and $0.295 \pm 0.030 \mathrm{cpm}$ (LC4, 5 and 6 , respectively). The weighted average, $0.276 \pm 0.016 \mathrm{cpm}$, is in excellent agreement with the directly measured $\Delta \mathrm{Ra}$ values averaging $0.279 \pm 0.026$.

The above analysis not only confirms the validity of the radon correction, but can also be used as a test of counting stability. For instance, for the four years, 1984-1987, the scatter standard deviation in the first- to last-day count differences, $\Delta \mathrm{Sa}, \Delta \mathrm{Ox}$ and $\Delta \mathrm{B}$ (Counter LC-5) are 0.297, $0.315,0.400$ and $0.371 \mathrm{cpm}$ for $\Delta \mathrm{Sa} ; 0.468,0.419,0.459$ and $0.372 \mathrm{cpm}$ for $\Delta \mathrm{Ox}$; and 0.090 , $0.075,0.072$, and $0.070 \mathrm{cpm}$ for $\Delta \mathrm{B}$. This gives a four-year average scatter standard deviation in the count differences of $0.346 \mathrm{cpm}$ for $\Delta \mathrm{Sa}, 0.430 \mathrm{cpm}$ for $\Delta \mathrm{Ox}$ and $0.077 \mathrm{cpm}$ for $\Delta \mathrm{B}$. The (average) total counts accumulated during a counting day yields a Poisson standard deviation in the difference of about $0.308 \mathrm{cpm}$ for $\Delta \mathrm{Sa}, 0.400 \mathrm{cpm}$ for $\Delta \mathrm{Ox}$ and $0.060 \mathrm{cpm}$ for $\Delta \mathrm{B}$. Thus, we 

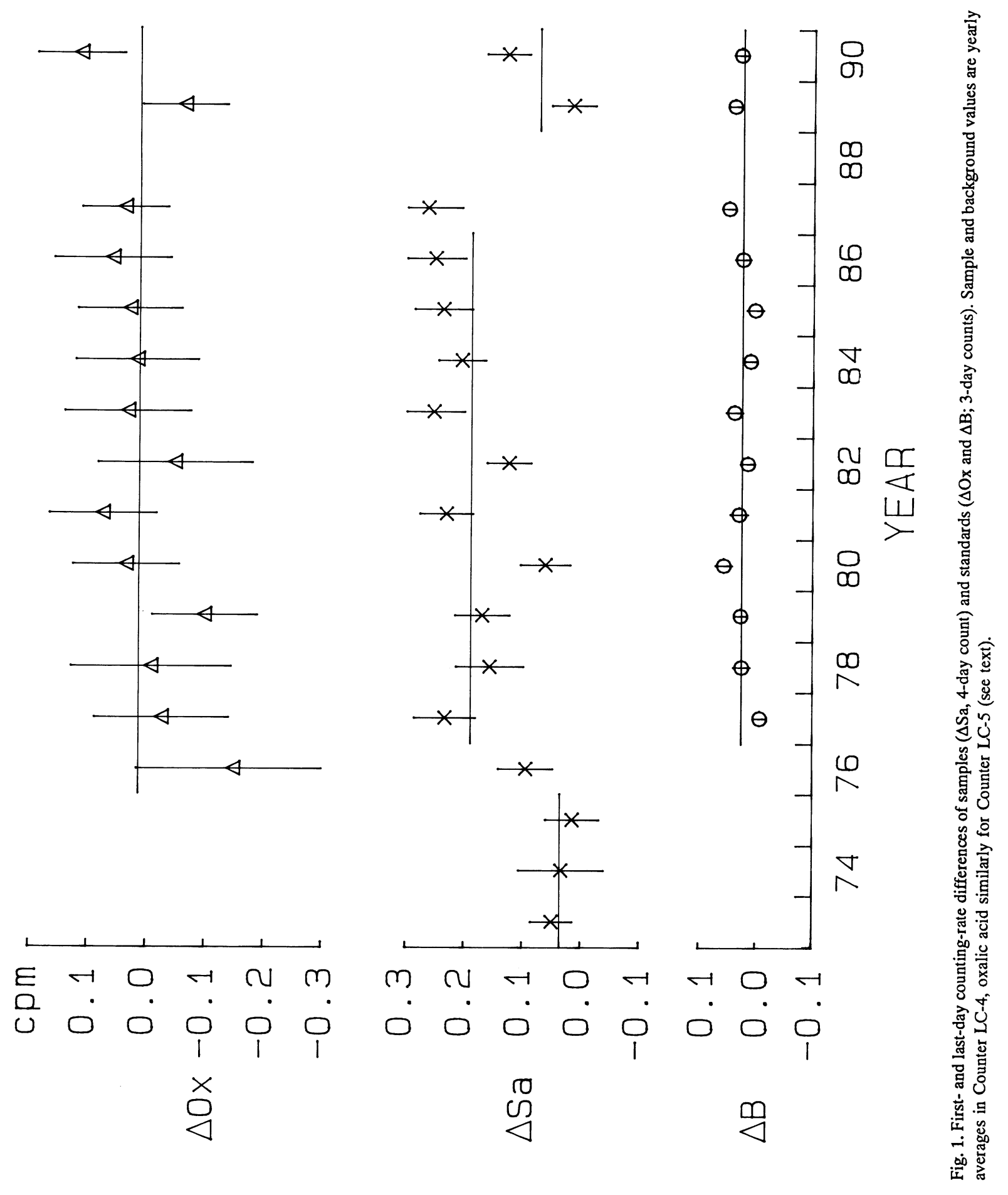
obtain error multipliers for the counting of samples and standards of $0.346 / 0.308=1.12\left(\mathrm{~K}_{\mathrm{sa}}\right)$, $0.430 / 0.400=1.08\left(\mathrm{~K}_{\mathrm{Ox}}\right)$ and $0.077 / 0.060=1.28\left(\mathrm{~K}_{\mathrm{B}}\right)$. Results for different years and different counters yield similar results, demonstrating excellent long-term counting stability.

The relative radon count-rate contribution, as a percentage of the observed sample counting rate, decreases when sample counts increase (younger samples). Correcting for the radon contribution results in an age increase of $10{ }^{14} \mathrm{C}$ yr for tree-ring samples that are a few hundred years old, and about $30{ }^{14} \mathrm{C}$ yr for $4500 \mathrm{yr}$-old samples.

\section{REPRODUCIBILITY AND SYSTEMATIC DIFFERENCES}

The ${ }^{14} \mathrm{C}$ ages reported are conventional ${ }^{14} \mathrm{C}$ ages (Stuiver \& Polach 1977). As noted in the introduction, the Poisson-derived standard deviation has been multiplied with an error multiplier, $\mathrm{K}$, of either 1.6 or 1.7. The justification of such a multiplier is given in Stuiver and Pearson (1992, 1993). The standard deviations shown in Table 1 reflect actual reproducibility. The $\mathrm{CO}_{2}$ gas proportional counters used for the previous study (Stuiver \& Becker 1986) also were used for the present one.

A comparison of Table 1 Seattle ${ }^{14} \mathrm{C}$ ages to those obtained by other laboratories confirms the validity of the choice of error multiplier. Systematic differences, although often confined to a decade or less, are more problematic. For the nearly 8000-yr Seattle and Belfast series (nearly 400 bidecadal data points), we find an average offset of $<1{ }^{14} \mathrm{C} \mathrm{yr}$, but this offset is not uniformly distributed. The offsets found for individual millennia are in the $0-17{ }^{14} \mathrm{C}$-yr range, with the exception of the $5180-5500 \mathrm{BC}$ interval, where the ${ }^{14} \mathrm{C}$ ages differ by an unacceptable $54 \pm 5 \mathrm{yr}$ (Stuiver \& Pearson 1993). Seattle and Heidelberg (Kromer et al. 1986) results differ by $41 \pm 4 \mathrm{yr}$ for the 4075-5265 BC and 5805-5995 BC intervals. The reasons for these offsets in the 40-50 yr range are not clear. Several other comparisons, e.g., with Groningen (3210-3910 BC), Tucson (5680-5810 BC), La Jolla (2500-5000 BC), Pretoria (1930-3350 BC) imply the lack of statistically significant offsets (Stuiver\& Pearson 1993). It is fair to conclude that systematic offsets in Table 1 are most likely confined to 1 or 2 decades, except for the 5180-5500 BC interval, where an offset up to $54 \mathrm{yr}$ is possible.

\section{CALIBRATION INSTRUCTIONS}

We recommend that users of ${ }^{14} \mathrm{C}$ dates obtain additional information on reproducibility (and systematic error, if any) from the laboratory reporting the ${ }^{14} \mathrm{C}$ date. This information should lead to a realistic standard deviation in the reported age. A systematic error has to be deducted from, or added to, the reported ${ }^{14} \mathrm{C}$ age prior to age calibration.

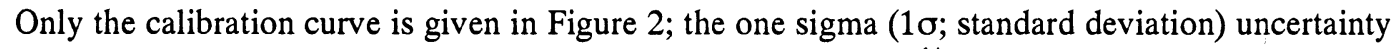
in the curve is not given. The standard deviation (averaging $24{ }^{14} \mathrm{C}$ yr) is tabulated in Table 1 for each decadal midpoint. Table 1 standard deviations reflect the entire variance in the age determination process.

Cal BP ages are relative to the year $\mathrm{AD} 1950$, with 0 cal BP equal to $\mathrm{AD} 1950$. The relationship between cal $\mathrm{AD} / \mathrm{BC}$ and Cal $\mathrm{BP}$ ages is cal $\mathrm{BP}=1950-\mathrm{cal} \mathrm{AD}$, and cal $\mathrm{BP}=1949+\mathrm{cal} \mathrm{BC}$ : The switch from 1950 to 1949 when converting $B C$ ages is caused by the absence of the year zero in the $\mathrm{AD} / \mathrm{BC}$ chronology.

The conversion of a ${ }^{14} \mathrm{C}$ age to a cal age is as follows: 1) draw line $\mathrm{A}$ parallel to the bottom axis through the ${ }^{14} \mathrm{C}$ age to be converted; 2 ) draw vertical line(s) through the intercept(s) of line $\mathrm{A}$ and 
the calibration curve. The cal $\mathrm{AD} / \mathrm{BC}$ ages can be read at the bottom axis, the cal $\mathrm{BP}$ ages at the top.

To convert the standard error in the ${ }^{14} \mathrm{C}$ age into a range of cal $\mathrm{AD} / \mathrm{BC}(\mathrm{BP})$ ages, determine the sample standard deviation, $\sigma$, by multiplying the quoted laboratory standard deviation with the "error multiplier". Unfortunately, information on error multipliers is often lacking. Here, the ${ }^{14} \mathrm{C}$ age user should refer to K values given above, or to the Scott, Long and Kra (1990).

Once the sample $\sigma$ is known, the curve $\sigma$ should be read from Table 1 . The curve $\sigma$ and sample $\sigma$ should then be used to calculate total $\sigma=\left((\text { sample } \sigma)^{2}+(\text { curve } \sigma)^{2}\right)^{1 / 2}$ (Stuiver 1982). Lines parallel to A should now be drawn through the ${ }^{14} \mathrm{C}$ age + total $\sigma$, and ${ }^{14} \mathrm{C}$ age - total $\sigma$ value. The vertical lines drawn through the intercepts now yield the outer limits of possible cal $\mathrm{AD} / \mathrm{BC}$ (cal BP) ages that are compatible with the sample standard deviation.

The conversion procedure yields 1) single or multiple cal $\mathrm{AD} / \mathrm{BC}$ (BP) ages that are compatible with a certain ${ }^{14} \mathrm{C}$ age, and 2) the range(s) of cal ages that correspond(s) to the standard deviation in the ${ }^{14} \mathrm{C}$ age (and calibration curve). Here, the user has to determine the calibrated ages from the Figure 2 graphs by drawing lines, whereas an alternate approach would be to use the computerized calibration (CALIB 3.0) program discussed elsewhere in this issue (Stuiver \& Reimer 1993).

The probability that a certain cal age is the actual sample age may be quite variable within the cal age range. Higher probabilities are encountered around the intercept ages. The non-linear transform of a near-Gaussian distribution around a ${ }^{14} \mathrm{C}$ age into cal $\mathrm{AD} / \mathrm{BC}$ (cal BP) age is not a simple matter, and computer programs are needed to derive the complex probability distribution. The CALIB 3.0 program (Stuiver \& Reimer 1993) incorporates such probability distributions.

The calibration data presented here are valid for northern hemispheric samples that were formed in equilibrium with atmospheric ${ }^{14} \mathrm{CO}_{2}$. Systematic age differences are possible for the southern hemisphere, where ${ }^{14} \mathrm{C}$ ages of wood samples tend to be about $40 \mathrm{yr}$ older (Vogel et al. 1993). Thus, ${ }^{14} \mathrm{C}$ ages of southern hemispheric samples preceding our era of fossil-fuel combustion should be reduced by $40 \mathrm{yr}$ before being converted into cal $\mathrm{AD} / \mathrm{BC}(\mathrm{BP})$ ages.

The calibration curve is valid only for age conversion of samples that were formed in equilibrium with atmospheric $\mathrm{CO}_{2}$. Conventional ${ }^{14} \mathrm{C}$ ages of materials not in equilibrium with atmospheric reservoirs do not take into account the offset in ${ }^{14} \mathrm{C}$ age that may occur (Stuiver \& Polach 1977). A constant offset, or reservoir deficiency, must be deducted from the reported ${ }^{14} \mathrm{C}$ age before any attempt can be made to convert to cal $\mathrm{AD} / \mathrm{BC}(\mathrm{BP})$ ages.

The reservoir deficiency is time dependent for the mixed (and deep) layer of the ocean. For the calibration of marine Holocene samples, the reader is referred to Stuiver and Braziunas (1993), and, of course, the CALIB 3.0 program.

\section{ACKNOWLEDGMENTS}

The ${ }^{14} \mathrm{C}$ research at Seattle was supported by National Science Foundation grant BNS-9004492. We thank P. J. Reimer and P. J. Wilkinson for crucial technical and analytical support. 


\section{REFERENCES}

Becker, B. 1993 An 11,000-year German oak and pine dendrochronology for radiocarbon calibration. Radiocarbon, this issue.

Ferguson, C. W. and Graybill, D. A. 1983 Dendrochronology of bristlecone pine: A progress report. In Stuiver, M. and Kra, R. S., Proceedings of the 11 th International Radiocarbon Conference. Radiocarbon 25(2): 287-288.

Kromer, B., Rhein, M., Bruns, M., Schoch-Fischer, H., Münnich, K. O., Stuiver, M. and Becker, B. 1986 Radiocarbon calibration data for the 6 th to the 8 th Millennia BC. In Stuiver, M. and Kra, R. S., eds., Proceedings of the 12th International Radiocarbon Conference. Radiocarbon 28(2B): 954-960.

Pearson, G. W. and Stuiver, M. 1993 High-precision bidecadal calibration of the radiocarbon time scale, 500-2500 BC. Radiocarbon, this issue.

Pilcher, J. R., Baillie, M. G. L., Schmidt, B. and Becker, B. 1984 A 7,272-year tree-ring chronology for western Europe. Nature 312: 150-152.

Scott, E. M., Long, A. and Kra, R., eds. 1990 Proceedings of the International Workshop on Intercomparison of Radiocarbon Laboratories. Radiocarbon 32(3): 253-397.

Stuiver, M. 1982 A high-precision calibration of the AD radiocarbon time scale. Radiocarbon 24(1): 1-26.

Stuiver, M. and Becker, B. 1986 High-precision decadal calibration of the radiocarbon time scale, AD 19502500 BC. In Stuiver, M. and Kra, R. S., eds., Proceedings of the 12th International Radiocarbon Conference. Radiocarbon 28(2B): 863-910.

Stuiver, M. and Braziunas, T. F. 1993 Modeling radiocarbon ages of marine samples back to $10,000 \mathrm{BC}$. Radiocarbon, this issue.

Stuiver, M. and Pearson, G. W. 1992 Calibration of the radiocarbon time scale, 2500-5000 BC. In Taylor, R. E., Long, A. and Kra, R. S., eds., Radiocarbon After Four Decades: An Interdisciplinary Perspective. New York, Springer Verlag: 19-33.

1993 High-precision calibration of the radiocarbon time scale, AD 1950-500 BC and 2500-6000 BC. Radiocarbon, this issue.

Stuiver, M. and Polach, H. A. 1977 Discussion: Reporting of ${ }^{14} \mathrm{C}$ data. Radiocarbon 19(3): 355-363.

Stuiver, M. and Reimer, P.J. 1993 Extended ${ }^{14} \mathrm{C}$ data base and revised CALIB radiocarbon age calibration program. Radiocarbon, this issue.

Vogel, J.C., Fuls, A., Visser, E. and Becker, B. 1993 Pretoria calibration curve for short-lived samples, 1930 BC-3350 BC. Radiocarbon, this issue. 


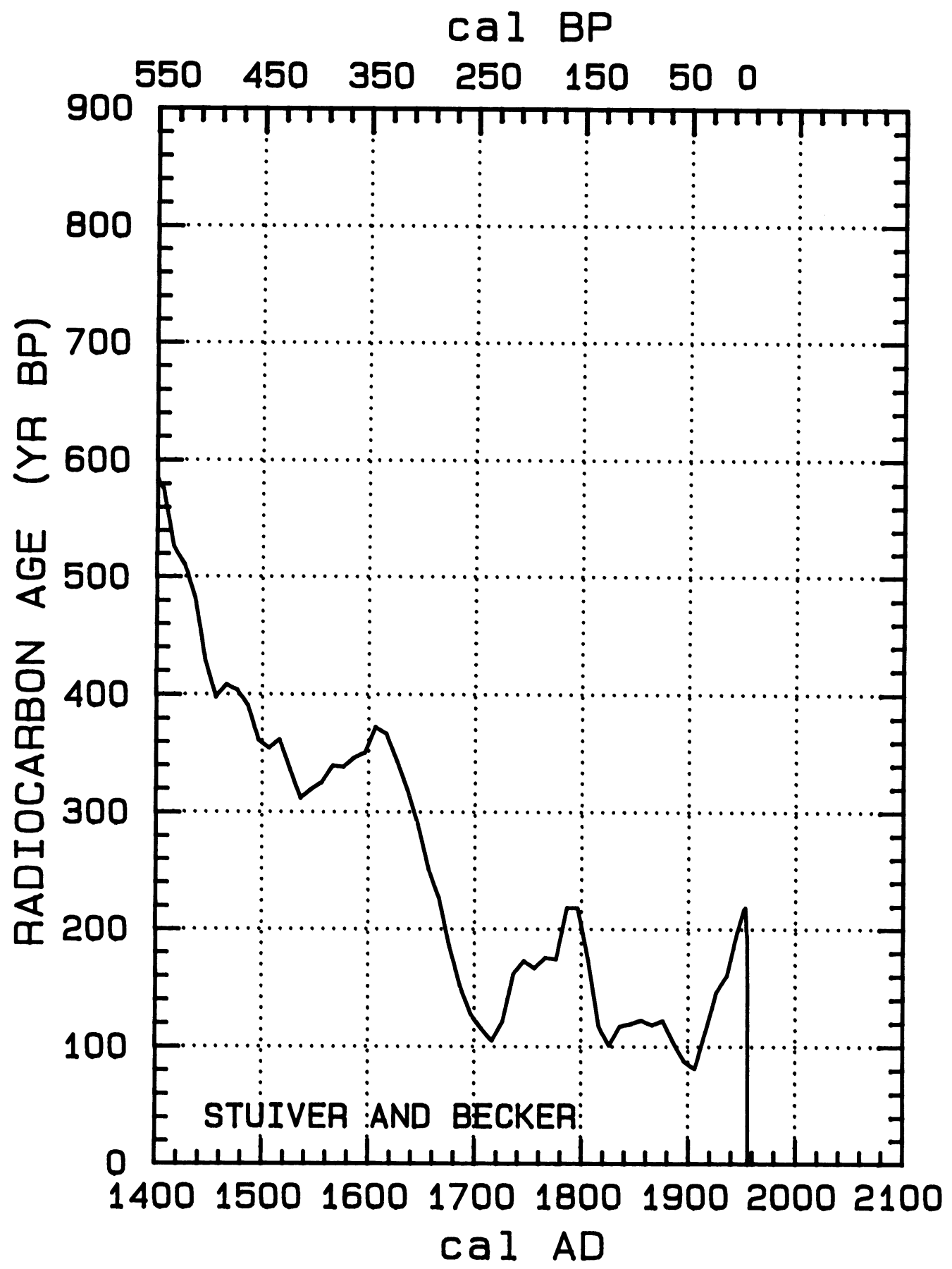

Fig. 2A-P. ${ }^{14} \mathrm{C}$ calibration curve derived from decadal samples 


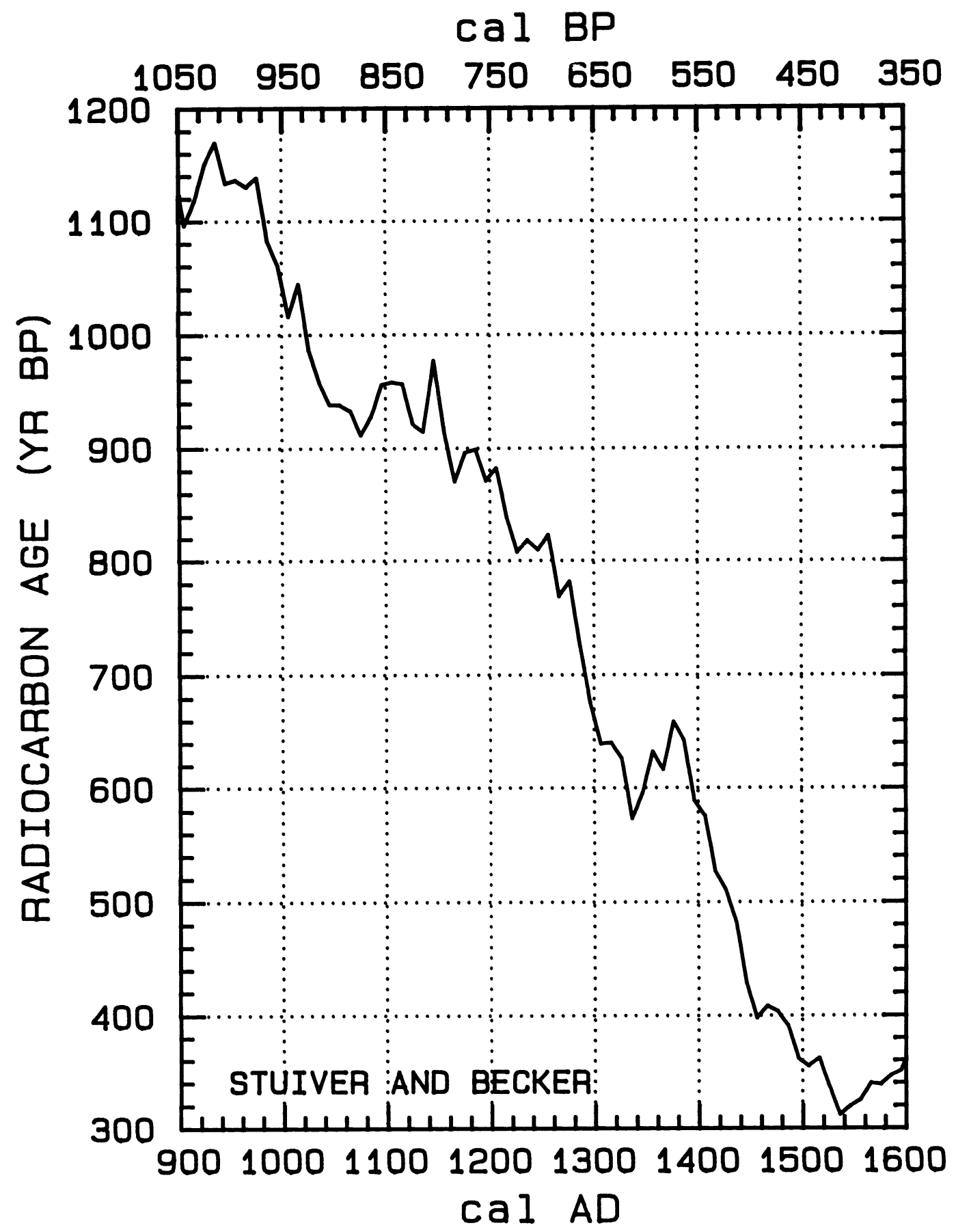

Fig. 2B 
cal BP

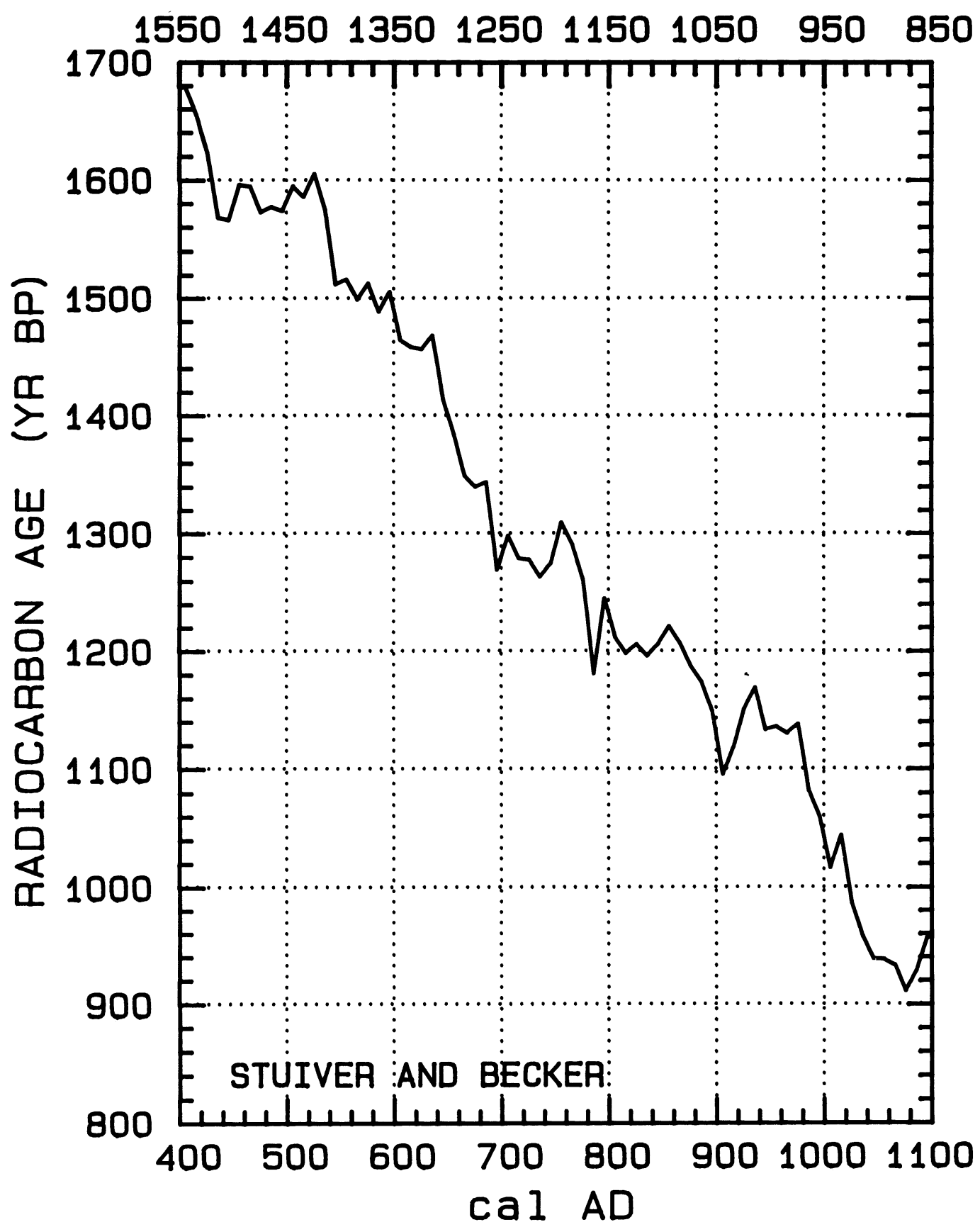

Fig. 2C 


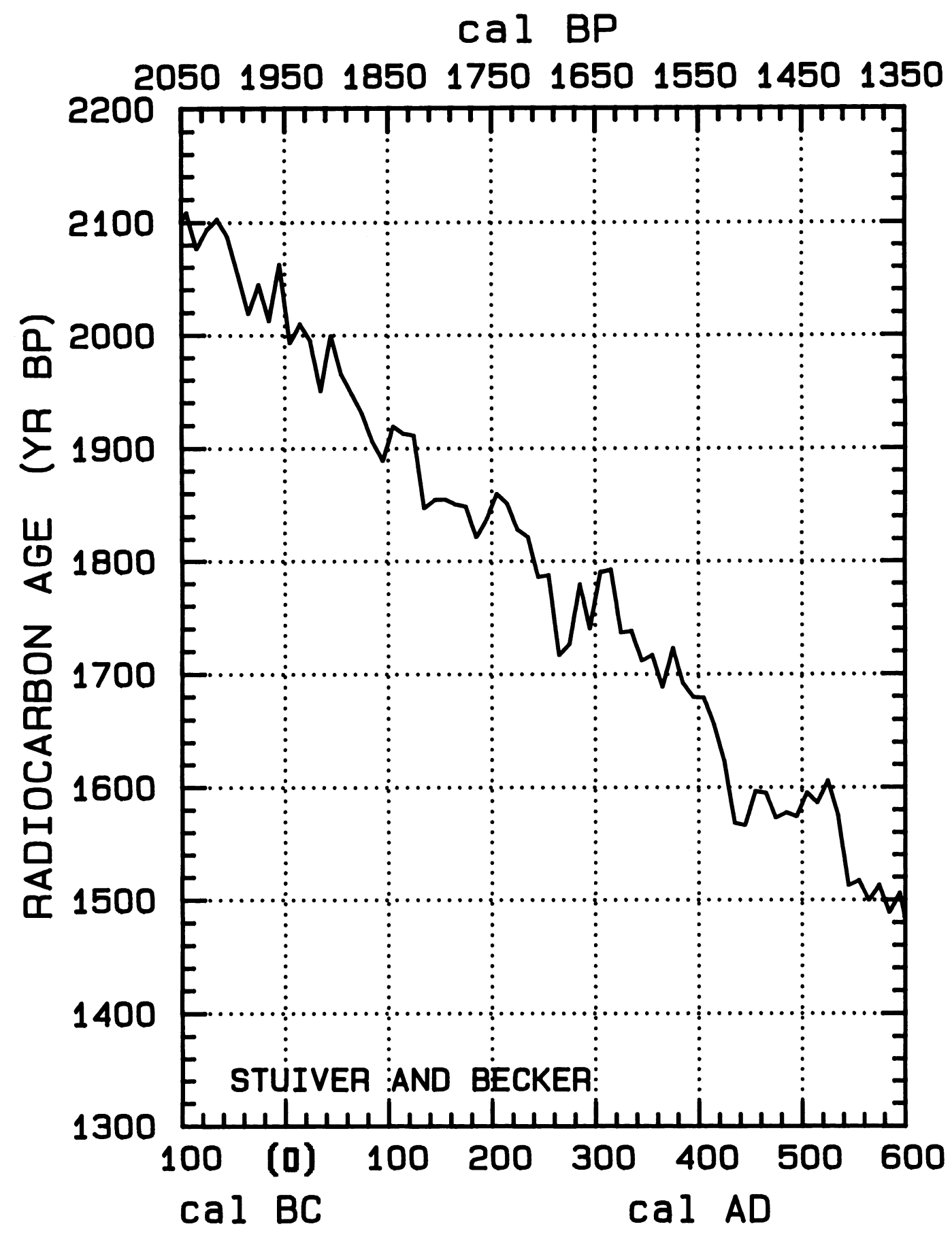

Fig. 2D 
cal BP

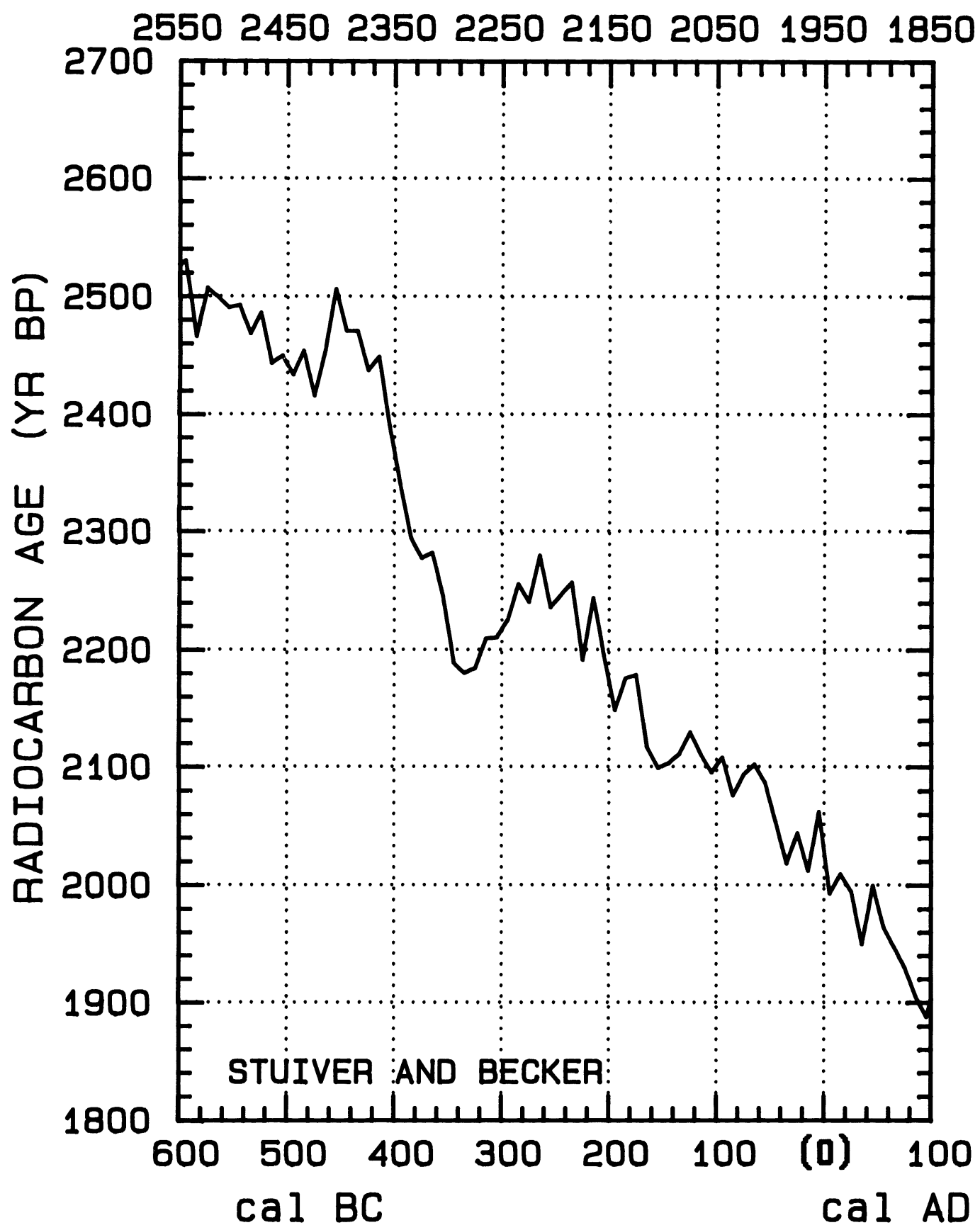

Fig. 2E 


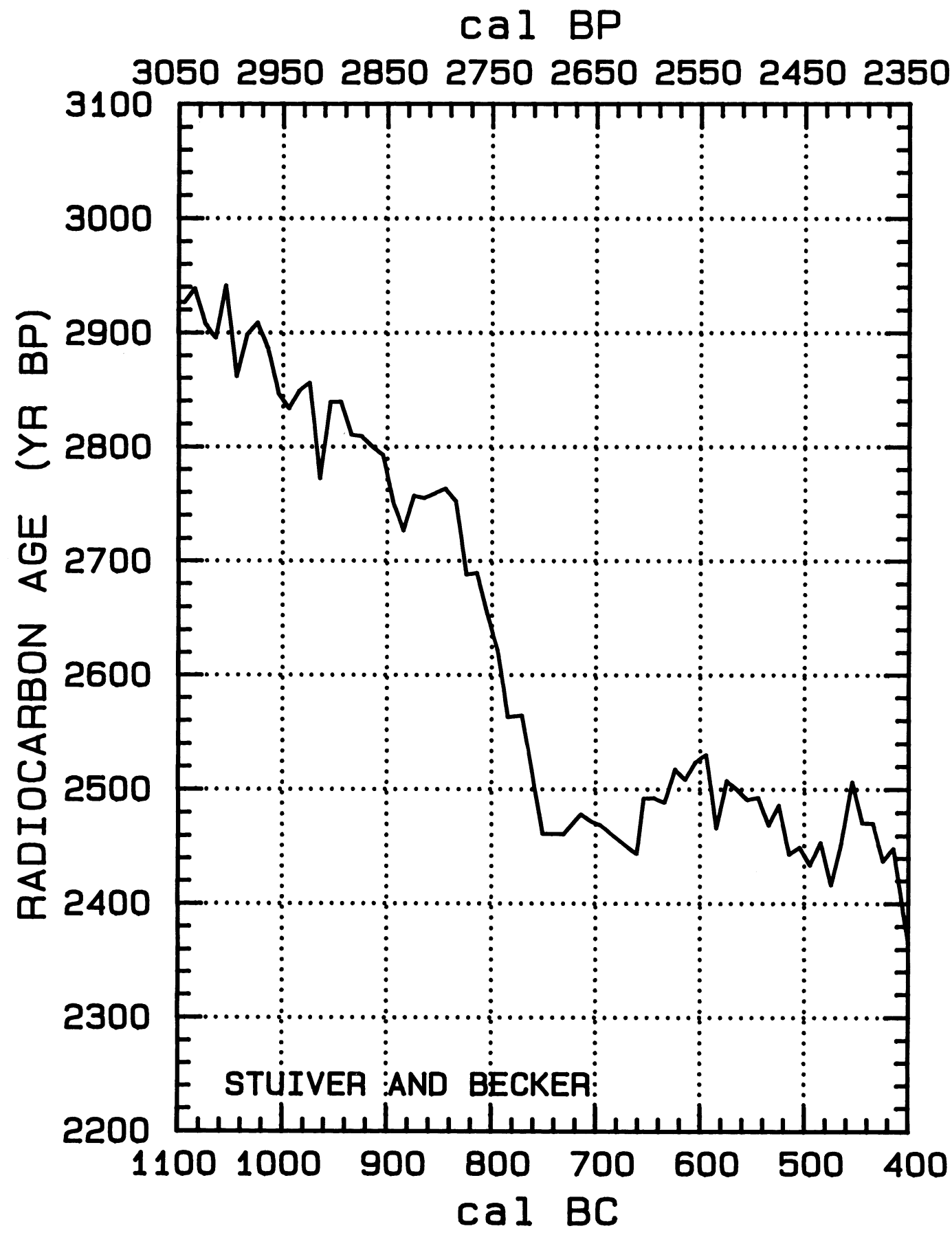

Fig. 2F 


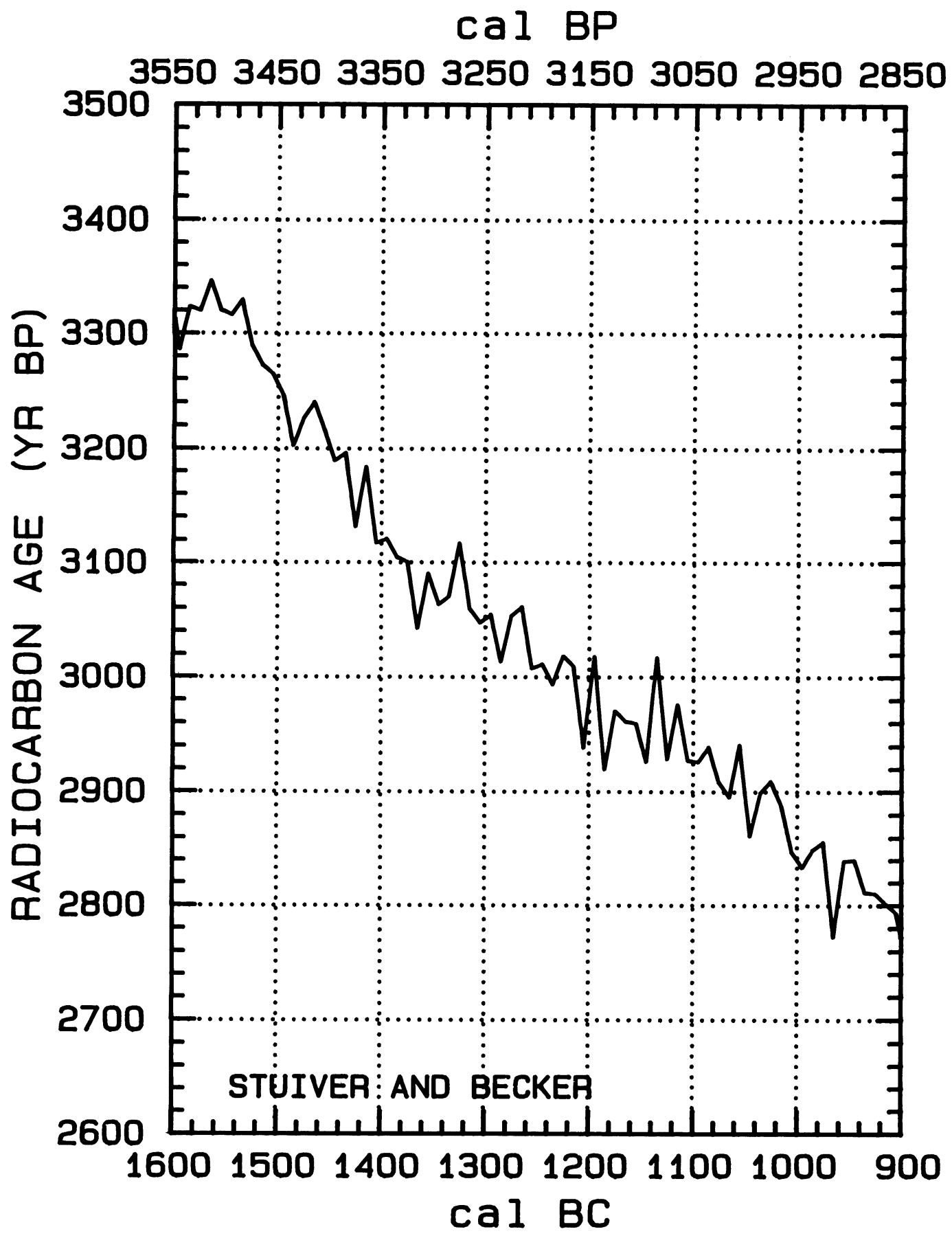

Fig. 2G 


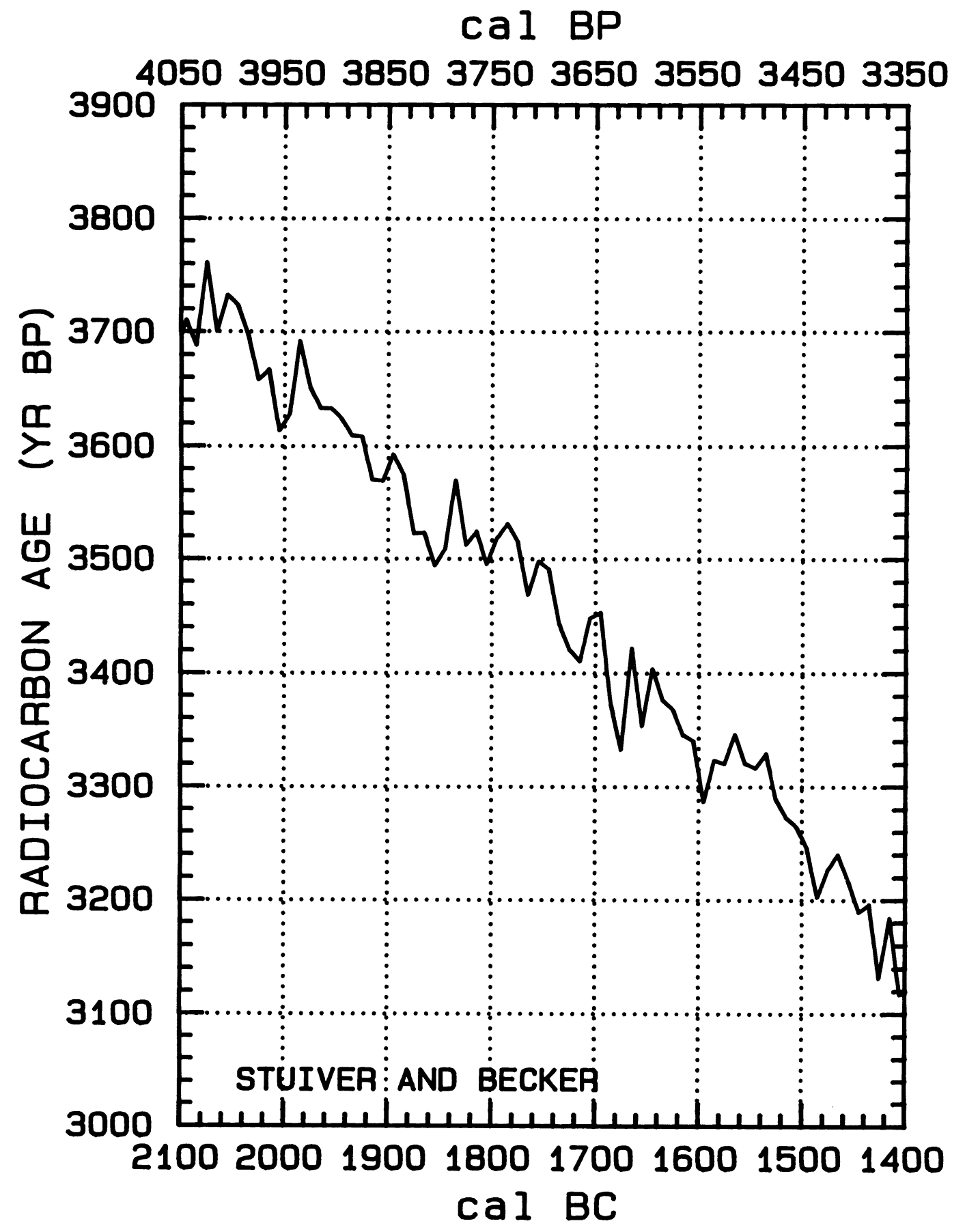

Fig. $2 \mathrm{H}$ 


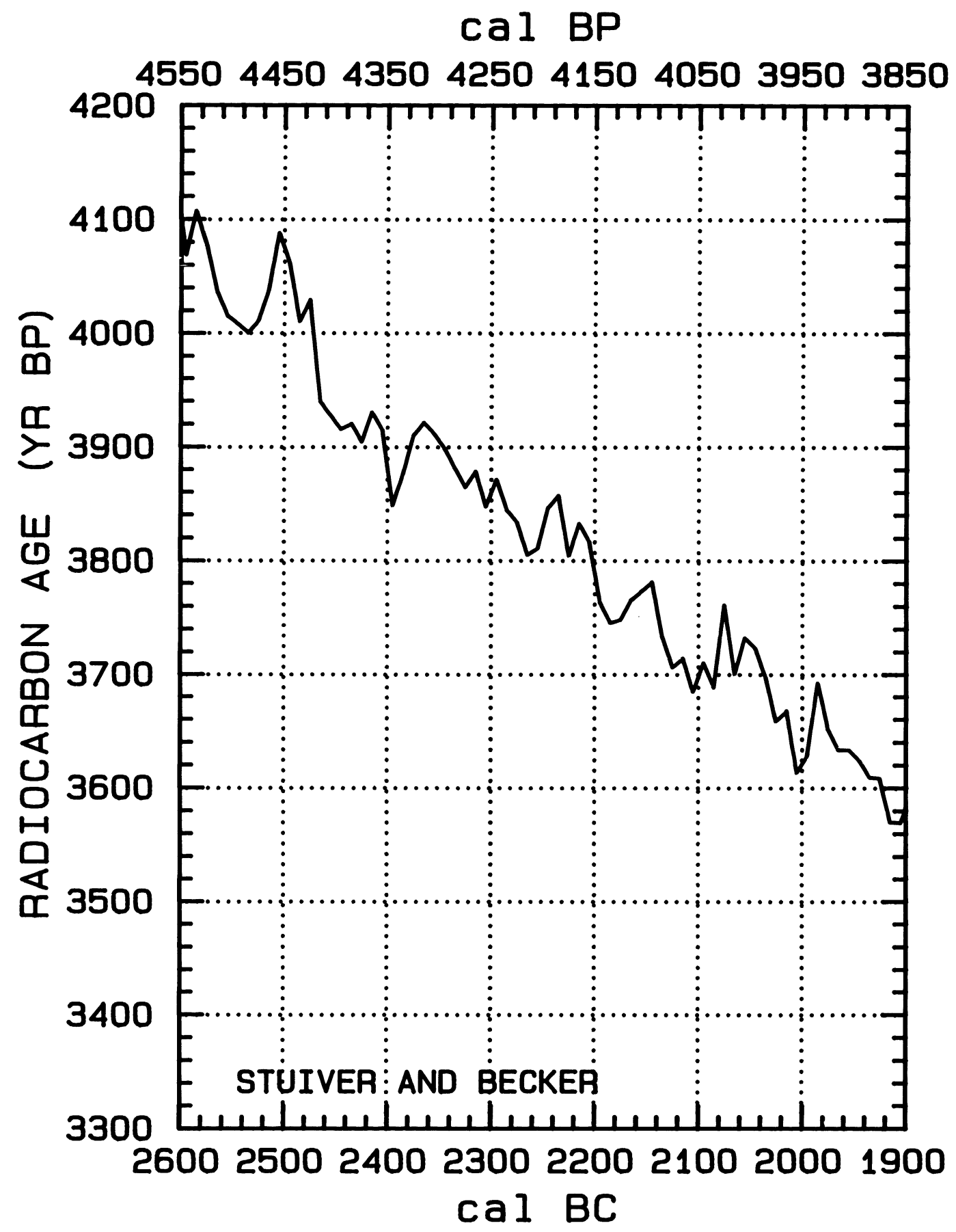

Fig. 2I 


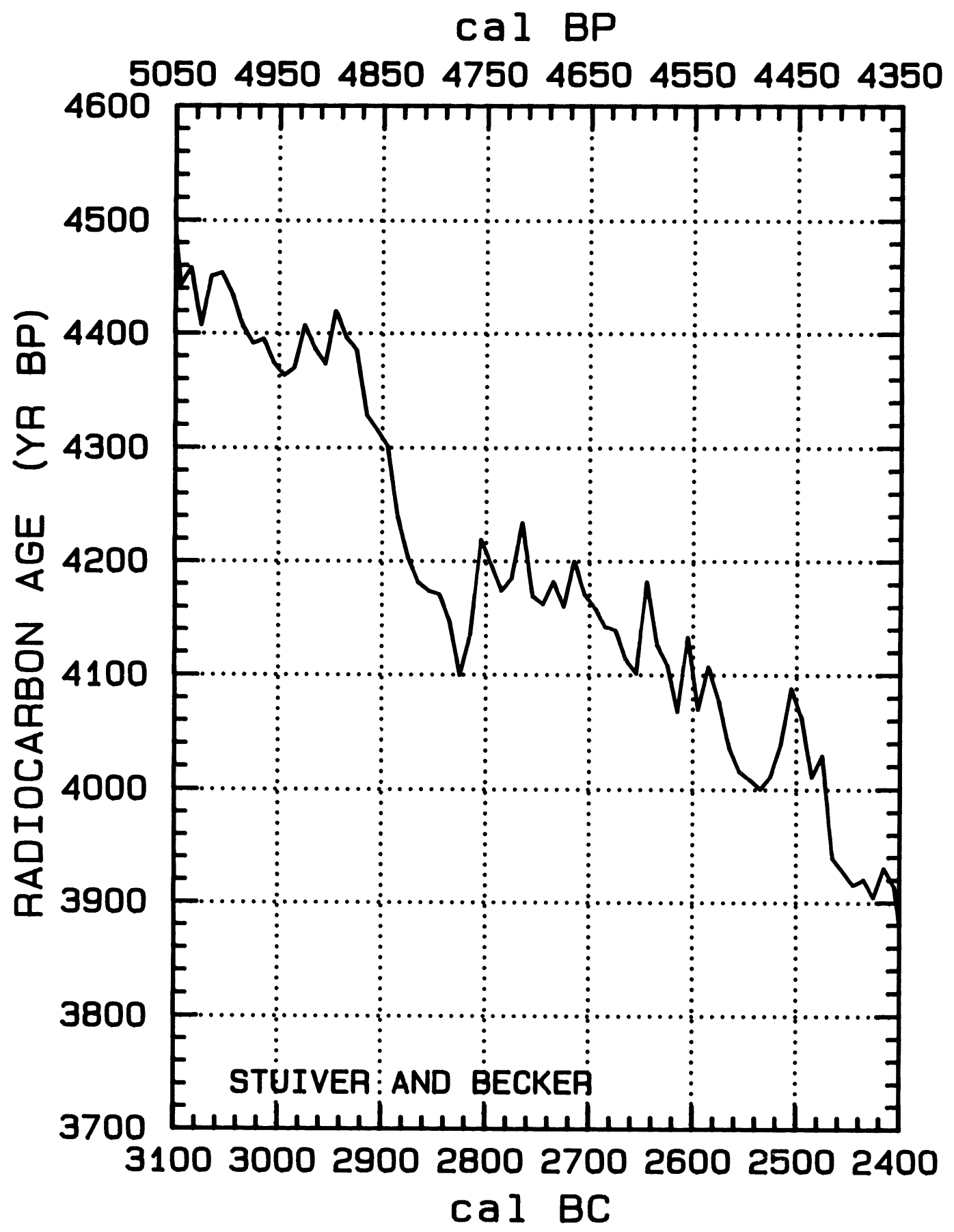

Fig. 2J 
cal BP

55505450535052505150505049504850

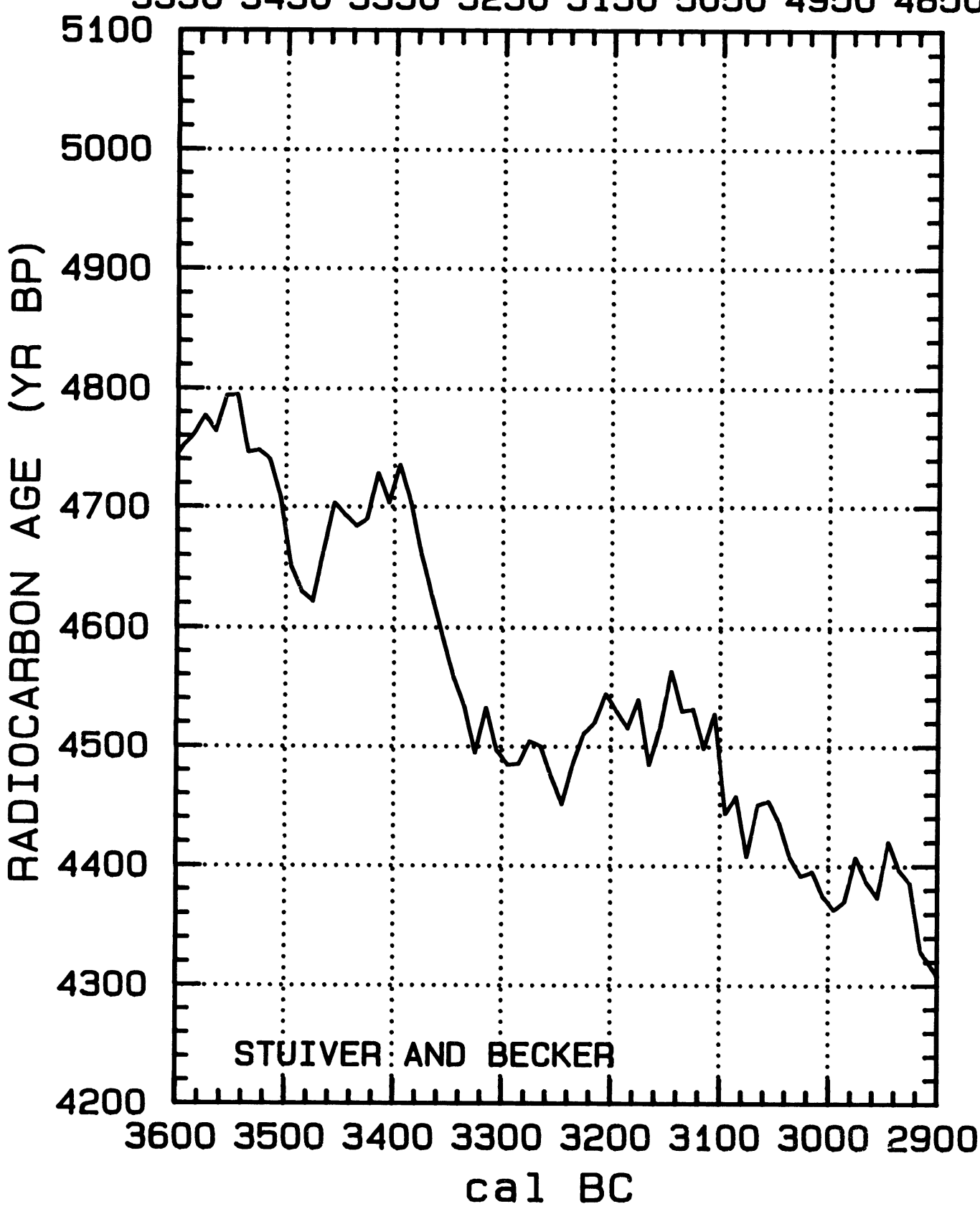

Fig. 2K 


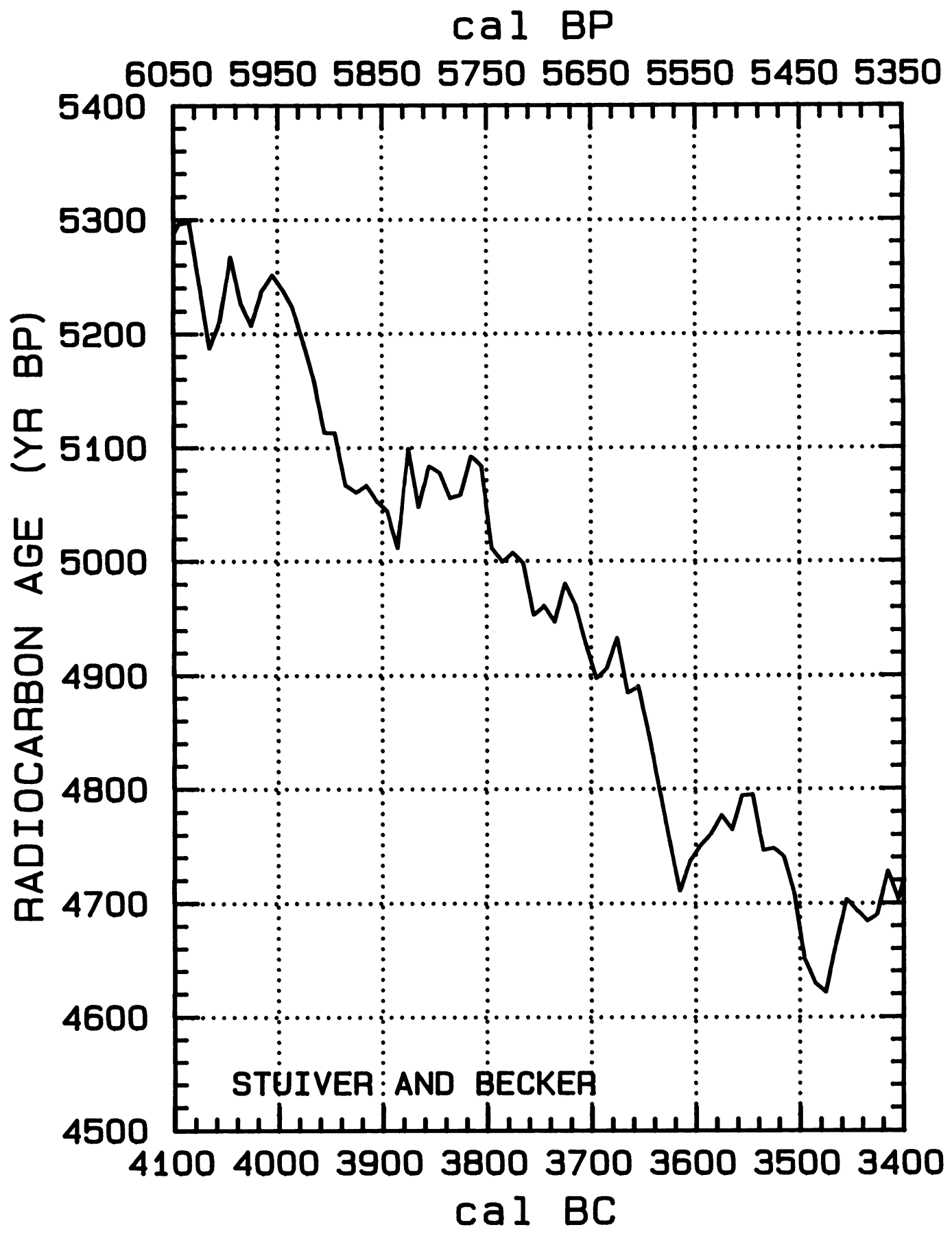

Fig. 2L 


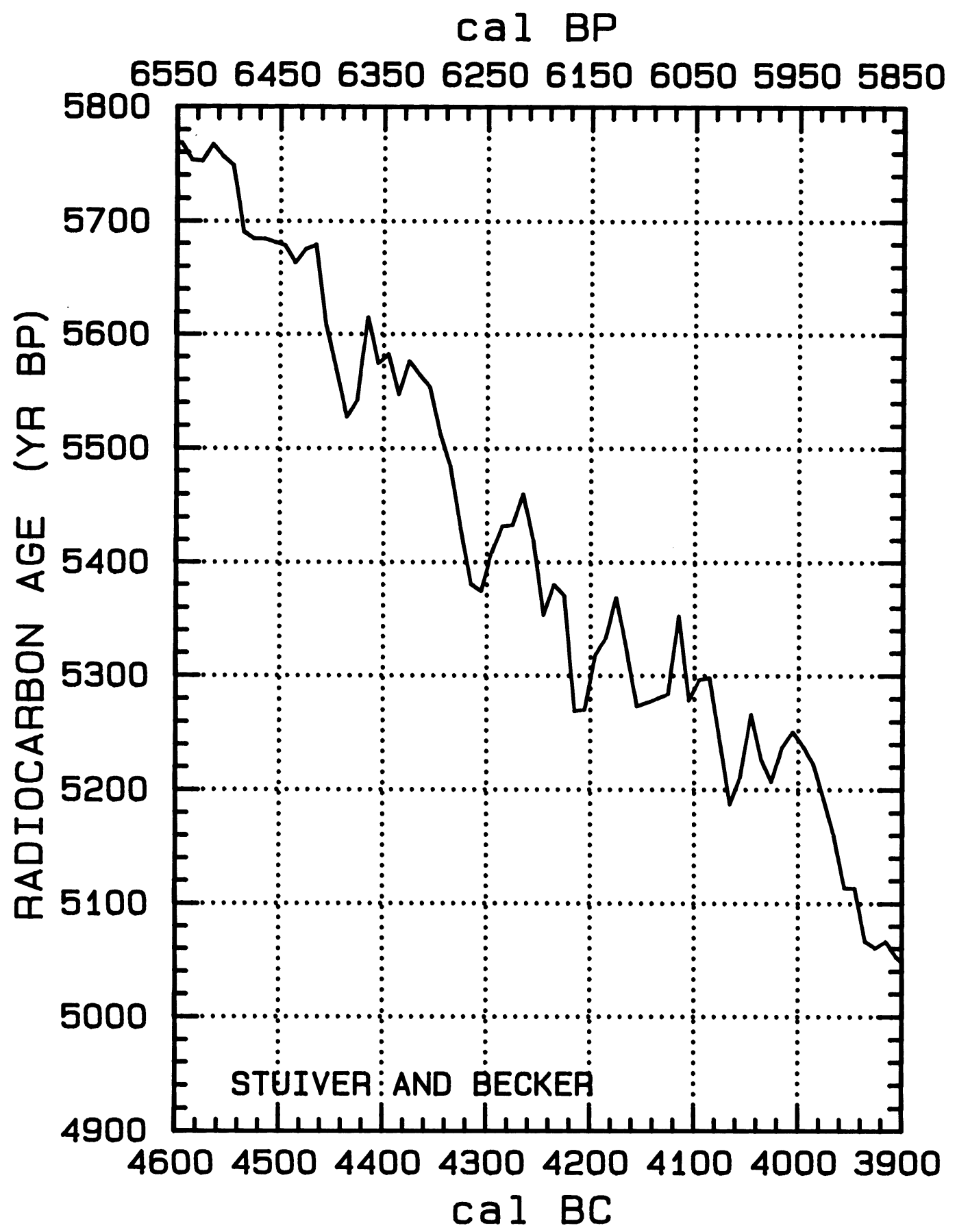

Fig. 2M 


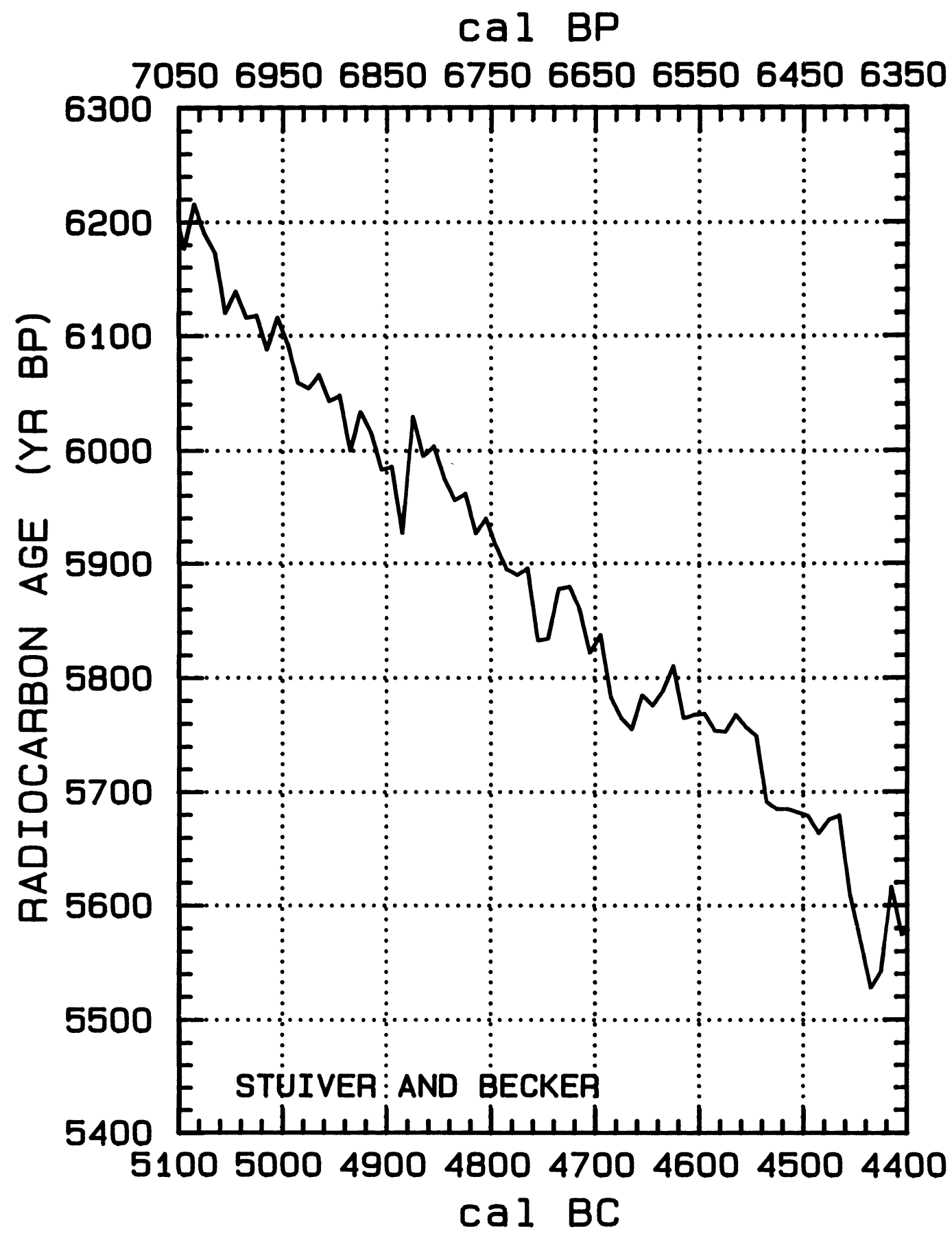

Fig. 2N 

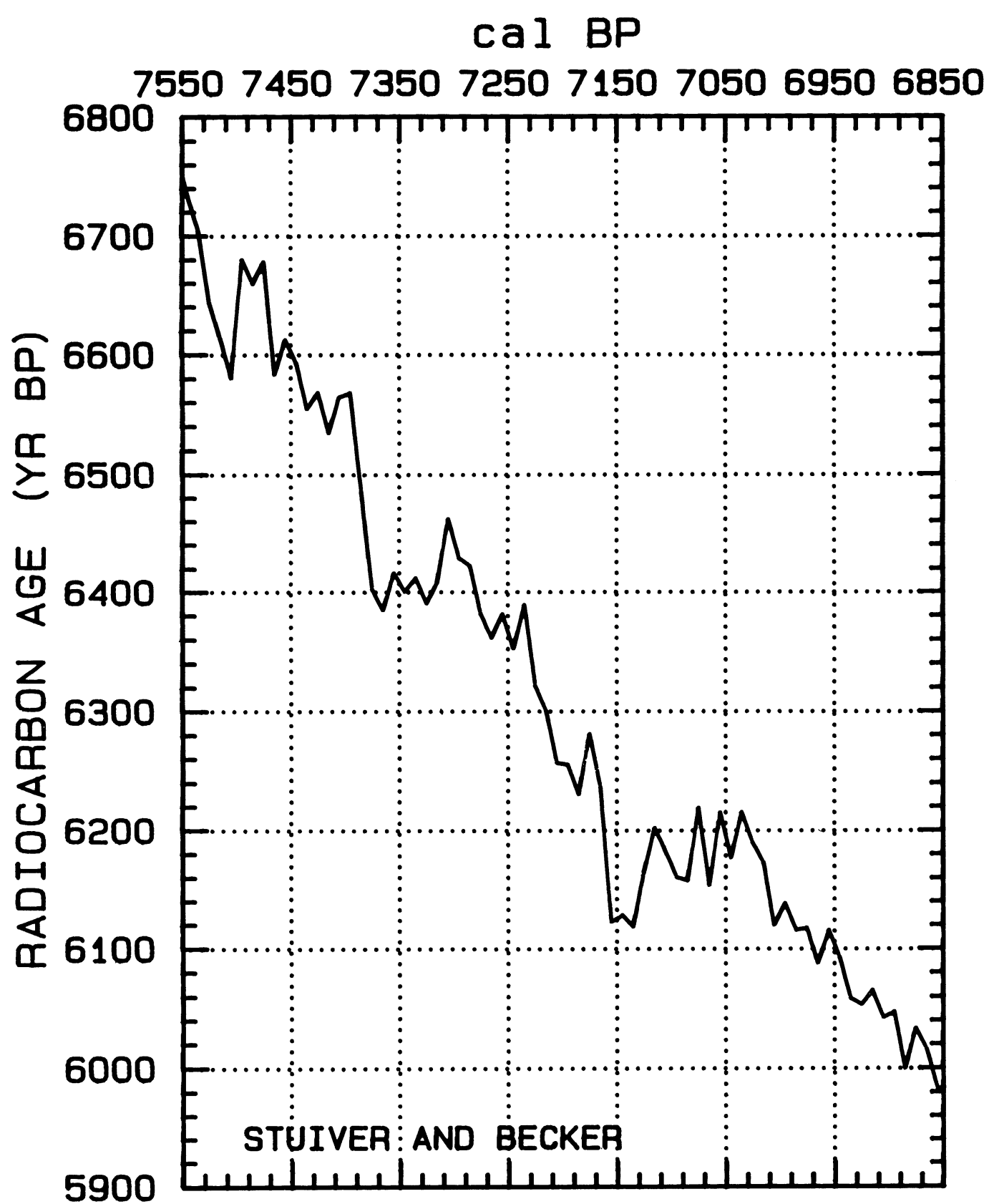

56005500540053005200510050004900

cal BC

Fig. 20 


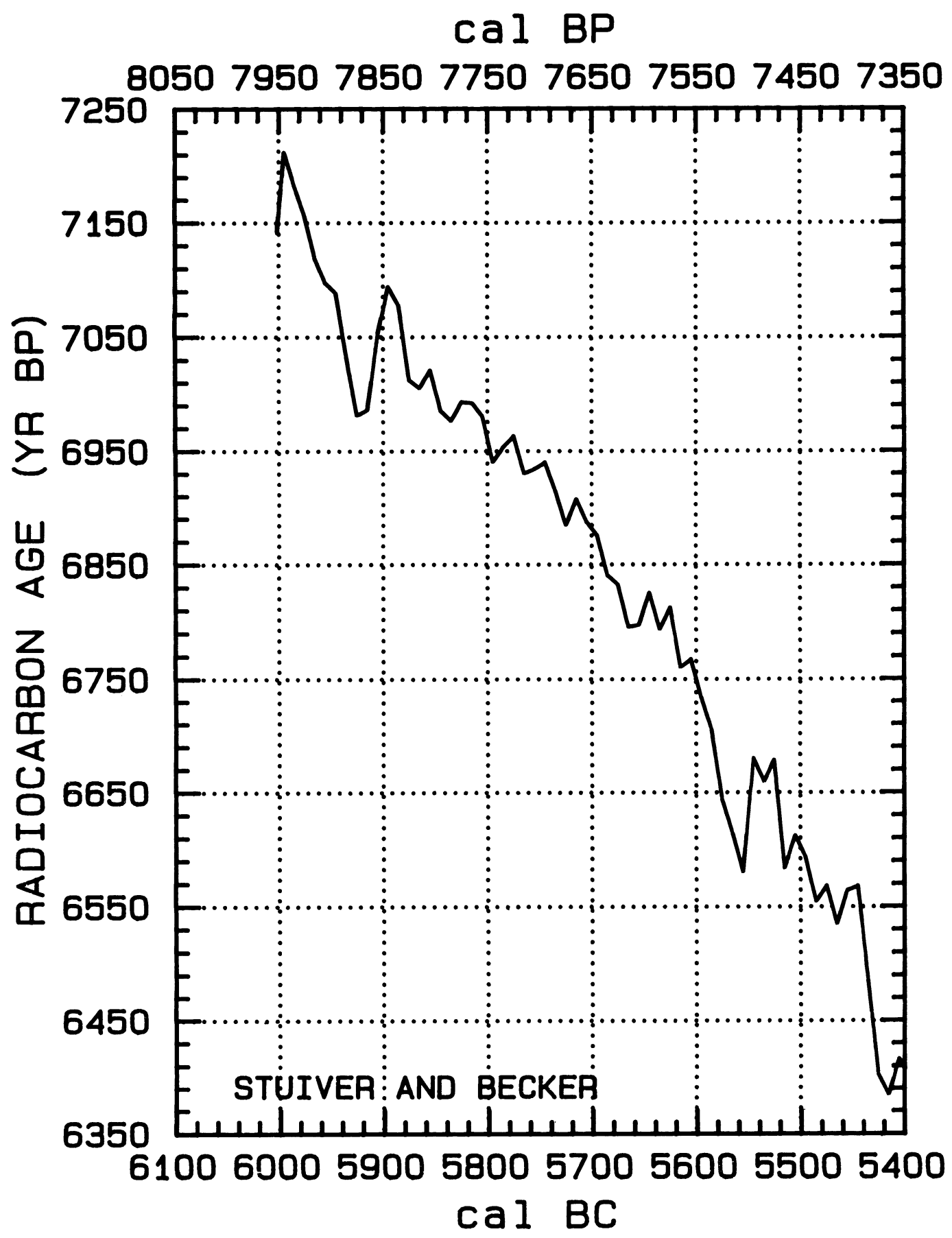

Fig. 2P 
TABLE $1 .{ }^{14} \mathrm{C}$ age determinations made at the University of Washington (Seattle). The cal $\mathrm{AD} / \mathrm{BC}$ ages (or cal BP) represent the midpoints of 10 -yr wood sections, except when 20 -yr samples were needed to obtain the quantity of treated wood used for a measurement $(661.5,696.5,731.5,751.5$ and $771.5 \mathrm{BC}$ ). The standard deviation in the age and $\Delta^{14} \mathrm{C}$ (defined in Stuiver \& Polach (1977)) values includes a 1.6 or 1.7 error multiplier (see text). Overlapping decadal samples with midpoints no greater than $1 \mathrm{yr}$ apart were averaged. Single-year data were averaged with decadal data for the AD 1515-1935 interval, and only single-year data were used for the AD 1945 data point.

\begin{tabular}{|c|c|c|c|c|c|c|c|}
\hline Cal $\mathrm{AD} / \mathrm{BC}$ & $\Delta^{14} \mathrm{C} \% 0$ & $\begin{array}{c}{ }^{14} \mathrm{C} \\
\text { age (BP) }\end{array}$ & Cal BP & $\mathrm{Cal} \mathrm{AD} / \mathrm{BC}$ & $\Delta^{14} \mathrm{C} \%$ & $\begin{array}{l}{ }^{14} \mathrm{C} \\
\text { age (BP) }\end{array}$ & Cal BP \\
\hline AD 1945 & $-23.7 \pm .8$ & $199 \pm 7$ & 5 & $\mathrm{AD} 1535$ & $11.5 \pm .7$ & $312 \pm 5$ & 415 \\
\hline AD 1935 & $-18.0 \pm .8$ & $161 \pm 6$ & 15 & AD 1525 & $9.7 \pm .8$ & $336 \pm 7$ & 425 \\
\hline AD 1925 & $-15.0 \pm .8$ & $146 \pm 6$ & 25 & $\mathrm{AD} 1515$ & $7.7 \pm .9$ & $362 \pm 7$ & 435 \\
\hline AD 1915 & $-9.8 \pm .6$ & $113 \pm 5$ & 35 & AD 1505 & $9.8 \pm 1.6$ & $355 \pm 13$ & 445 \\
\hline AD 1905 & $-4.7 \pm 1.0$ & $81 \pm 8$ & 45 & AD 1495 & $10.1 \pm 2.0$ & $362 \pm 16$ & 455 \\
\hline AD 1895 & $-4.2 \pm 1.1$ & $88 \pm 9$ & 55 & AD 1485 & $7.6 \pm 2.6$ & $392 \pm 21$ & 465 \\
\hline $\mathrm{AD} 1885$ & $-5.0 \pm .7$ & $104 \pm 5$ & 65 & $\mathrm{AD} 1475$ & $7.2 \pm 2.8$ & $404 \pm 23$ & 475 \\
\hline AD 1875 & $-6.1 \pm .7$ & $122 \pm 5$ & 75 & $\mathrm{AD} 1465$ & $7.9 \pm 2.9$ & $409 \pm 23$ & 485 \\
\hline $\mathrm{AD} 1865$ & $-4.5 \pm .7$ & $119 \pm 6$ & 85 & $\mathrm{AD} 1455$ & $10.5 \pm 2.0$ & $398 \pm 16$ & 495 \\
\hline $\mathrm{AD} 1855$ & $-3.9 \pm .9$ & $123 \pm 8$ & 95 & $\mathrm{AD} 1445$ & $7.9 \pm 2.4$ & $428 \pm 19$ & 505 \\
\hline $\mathrm{AD} 1845$ & $-2.1 \pm .8$ & $120 \pm 6$ & 105 & AD 1435 & $2.3 \pm 2.9$ & $483 \pm 23$ & 515 \\
\hline AD 1835 & $-0.7 \pm .7$ & $117 \pm 6$ & 115 & $\mathrm{AD} 1425$ & $0.0 \pm 2.7$ & $511 \pm 21$ & 525 \\
\hline AD 1825 & $2.6 \pm .6$ & $101 \pm 5$ & 125 & AD 1415 & $-0.8 \pm 2.8$ & $527 \pm 22$ & 535 \\
\hline AD 1815 & $1.7 \pm .8$ & $118 \pm 7$ & 135 & AD 1405 & $-5.7 \pm 2.6$ & $576 \pm 21$ & 545 \\
\hline AD 1805 & $-3.9 \pm .8$ & $173 \pm 6$ & 145 & $\mathrm{AD} 1395$ & $-6.1 \pm 2.8$ & $589 \pm 23$ & 555 \\
\hline AD 1795 & $-8.4 \pm .8$ & $219 \pm 7$ & 155 & AD 1385 & $-11.6 \pm 2.0$ & $643 \pm 16$ & 565 \\
\hline AD 1785 & $-7.3 \pm .8$ & $219 \pm 7$ & 165 & AD 1375 & $-12.4 \pm 2.2$ & $659 \pm 18$ & 575 \\
\hline AD 1775 & $-0.9 \pm .7$ & $174 \pm 6$ & 175 & $\mathrm{AD} 1365$ & $-5.9 \pm 2.1$ & $616 \pm 17$ & 585 \\
\hline AD 1765 & $0.2 \pm .8$ & $175 \pm 6$ & 185 & $\mathrm{AD} 1355$ & $-6.7 \pm 2.3$ & $632 \pm 18$ & 595 \\
\hline AD 1755 & $2.6 \pm .6$ & $167 \pm 5$ & 195 & $\mathrm{AD} 1345$ & $-0.9 \pm 2.3$ & $596 \pm 19$ & 605 \\
\hline AD 1745 & $3.0 \pm .7$ & $173 \pm 6$ & 205 & $\mathrm{AD} 1335$ & $3.1 \pm 2.0$ & $573 \pm 16$ & 615 \\
\hline AD 1735 & $5.5 \pm .8$ & $161 \pm 6$ & 215 & AD 1325 & $-2.4 \pm 2.1$ & $627 \pm 17$ & 625 \\
\hline AD 1725 & $12.0 \pm .6$ & $122 \pm 5$ & 225 & AD 1315 & $-2.9 \pm 2.6$ & $641 \pm 21$ & 635 \\
\hline $\mathrm{AD} 1715$ & $15.2 \pm .5$ & $105 \pm 4$ & 235 & AD 1305 & $-1.6 \pm 1.6$ & $640 \pm 13$ & 645 \\
\hline AD 1705 & $15.2 \pm .5$ & $116 \pm 4$ & 245 & AD 1295 & $-4.9 \pm 2.9$ & $676 \pm 24$ & 655 \\
\hline $\mathrm{AD} 1695$ & $14.8 \pm .5$ & $129 \pm 4$ & 255 & $\mathrm{AD} 1285$ & $-9.7 \pm 2.7$ & $725 \pm 22$ & 665 \\
\hline $\mathrm{AD} 1685$ & $13.1 \pm .7$ & $152 \pm 6$ & 265 & $\mathrm{AD} 1275$ & $-15.5 \pm 2.9$ & $782 \pm 24$ & 675 \\
\hline $\mathrm{AD} 1675$ & $10.4 \pm .7$ & $185 \pm 6$ & 275 & $\mathrm{AD} 1265$ & $-12.7 \pm 2.9$ & $769 \pm 23$ & 685 \\
\hline AD 1665 & $6.3 \pm .7$ & $227 \pm 6$ & 285 & $\mathrm{AD} 1255$ & $-18.2 \pm 2.7$ & $823 \pm 22$ & 695 \\
\hline $\mathrm{AD} 1655$ & $4.5 \pm .8$ & $251 \pm 6$ & 295 & AD 1245 & $-15.4 \pm 2.8$ & $810 \pm 23$ & 705 \\
\hline AD 1645 & $0.9 \pm .7$ & $290 \pm 6$ & 305 & AD 1235 & $-15.2 \pm 2.8$ & $819 \pm 23$ & 715 \\
\hline AD 1635 & $-1.7 \pm .7$ & $320 \pm 6$ & 315 & AD 1225 & $-12.7 \pm 2.0$ & $808 \pm 16$ & 725 \\
\hline $\mathrm{AD} 1625$ & $-3.4 \pm .7$ & $344 \pm 6$ & 325 & AD 1215 & $-15.4 \pm 2.8$ & $839 \pm 23$ & 735 \\
\hline $\mathrm{AD} 1615$ & $-5.1 \pm .8$ & $367 \pm 6$ & 335 & AD 1205 & $-19.5 \pm 2.8$ & $883 \pm 23$ & 745 \\
\hline AD 1605 & $-4.6 \pm .7$ & $373 \pm 6$ & 345 & AD 1195 & $-16.9 \pm 2.8$ & $871 \pm 23$ & 755 \\
\hline AD 1595 & $-0.7 \pm .9$ & $351 \pm 7$ & 355 & AD 1185 & $-19.1 \pm 2.6$ & $899 \pm 21$ & 765 \\
\hline AD 1585 & $1.1 \pm .8$ & $346 \pm 7$ & 365 & $\mathrm{AD} 1175$ & $-17.6 \pm 2.8$ & $896 \pm 23$ & 775 \\
\hline AD 1575 & $3.3 \pm .9$ & $338 \pm 7$ & 375 & AD 1165 & $-13.2 \pm 2.0$ & $870 \pm 16$ & 785 \\
\hline $\mathrm{AD} 1565$ & $4.3 \pm .8$ & $340 \pm 6$ & 385 & AD 1155 & $-17.4 \pm 2.8$ & $914 \pm 23$ & 795 \\
\hline AD 1555 & $7.4 \pm .8$ & $325 \pm 6$ & 395 & AD 1145 & $-23.9 \pm 2.0$ & $977 \pm 16$ & 805 \\
\hline AD 1545 & $9.4 \pm .8$ & $319 \pm 6$ & 405 & AD 1135 & $-15.2 \pm 2.2$ & $915 \pm 18$ & 815 \\
\hline
\end{tabular}


TABLE 1. (Continued)

\begin{tabular}{|c|c|c|c|c|c|c|c|c|}
\hline \multirow[b]{2}{*}{ Cal $\mathrm{AD} / \mathrm{BC}$} & \multicolumn{3}{|c|}{${ }^{14} \mathrm{C}$} & \multirow{2}{*}{\multicolumn{2}{|c|}{$\mathrm{Cal} \mathrm{AD} / \mathrm{BC}$}} & \multicolumn{3}{|c|}{${ }^{14} \mathrm{C}$} \\
\hline & $\Delta^{14} \mathrm{C} \% 0$ & age (BP) & $\mathrm{Cal} \mathrm{BP}$ & & & $\Delta^{14} \mathrm{C} \% 0$ & age (BP) & $v_{1}$ \\
\hline AD 1125 & $-14.8 \pm 2.9$ & $922 \pm 24$ & 825 & $\overline{\mathrm{AD}}$ & 655 & $-15.5 \pm 3.0$ & $1384 \pm 25$ & 1295 \\
\hline D 1115 & $-17.9 \pm 2.1$ & $957 \pm 17$ & & $\mathrm{AD}$ & 645 & $-17.9 \pm 3.4$ & $1414 \pm 28$ & 1305 \\
\hline D 1105 & $-17.0 \pm 1.9$ & $958 \pm 16$ & 845 & $\mathrm{AD}$ & 635 & $-23.4 \pm 2.3$ & $1468 \pm 19$ & 1315 \\
\hline AD 1095 & $-15.5 \pm 2.1$ & $956 \pm 17$ & & $\mathrm{AD}$ & 625 & $-20.8 \pm 3.1$ & $1457 \pm 25$ & 1325 \\
\hline AD 1085 & \pm 1.5 & $928 \pm 12$ & & $\mathrm{AD}$ & 615 & \pm 6.2 & $1458 \pm 51$ & 1335 \\
\hline D 1075 & -7.6 & $911 \pm 12$ & 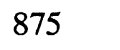 & $\mathrm{AD}$ & 605 & $-19.4 \pm 2.9$ & $1465 \pm 24$ & 1345 \\
\hline 1065 & \pm 1.7 & $933 \pm 14$ & 88 & $\mathrm{AD}$ & 595 & $-23.2 \pm 3.2$ & $1506 \pm 27$ & 1355 \\
\hline 1055 & \pm 1.8 & $938 \pm 15$ & 895 & $\mathrm{AD}$ & 585 & $-19.9 \pm 3.0$ & $1488 \pm 25$ & 1365 \\
\hline $\mathrm{AD} 1045$ & \pm 2.0 & $939 \pm 17$ & 905 & $\mathrm{AD}$ & 575 & $-21.7 \pm 3.4$ & $1513 \pm 28$ & 1375 \\
\hline $\mathrm{AD} 1035$ & $-8.6 \pm 2.0$ & $958 \pm 16$ & & $\mathrm{AD}$ & 565 & $3 \pm 3.2$ & $1499 \pm 26$ & 1385 \\
\hline $\mathrm{AD} 1025$ & -10 & $986 \pm 23$ & 92 & $\mathrm{AD}$ & 555 & $3 \pm 3.3$ & $1517 \pm 27$ & 1395 \\
\hline AD 1015 & & $1044 \pm 24$ & 93 & $\mathrm{AD}$ & 545 & $-18.1 \pm 2.2$ & $1512 \pm 18$ & 1405 \\
\hline AD 1005 & -12 & $1016 \pm 24$ & & $\mathrm{AD}$ & 535 & $-24.6 \pm 3.1$ & $1575 \pm 26$ & 1415 \\
\hline AD 995 & -16 & $1060 \pm 21$ & 95 & $\mathrm{AD}$ & 525 & \pm 1.9 & $1606 \pm 16$ & 1425 \\
\hline AD 985 & -17 & $1082 \pm 21$ & & $\mathrm{AD}$ & 515 & $-23.5 \pm 2.8$ & $1586 \pm 23$ & 435 \\
\hline AD 975 & -23 & $1138 \pm 24$ & & $\mathrm{AD}$ & 505 & \pm 2.9 & $1595 \pm 23$ & 445 \\
\hline $\mathrm{AD} \quad 965$ & 2.9 & $1130 \pm 24$ & & $\mathrm{AD}$ & 495 & \pm 2.6 & $1574 \pm 21$ & 455 \\
\hline $\mathrm{AD} \quad 955$ & 3.0 & $1136 \pm 25$ & 99 & $\mathrm{AD}$ & 485 & \pm 3.2 & $1577 \pm 27$ & 465 \\
\hline AD 945 & -15 & $1133 \pm 25$ & & $\mathrm{AD}$ & 475 & \pm 3.0 & $1573 \pm 25$ & 475 \\
\hline AD 935 & -22 & $1170 \pm 26$ & & $\mathrm{AD}$ & 465 & \pm 3.2 & $1595 \pm 26$ & 485 \\
\hline $\mathrm{AD} \quad 925$ & 2.1 & $1151 \pm 17$ & & $\mathrm{AD}$ & 455 & \pm 3.2 & $1596 \pm 26$ & 495 \\
\hline $\mathrm{AD} \quad 915$ & -13 & $1118 \pm 23$ & & $\mathrm{AD}$ & 445 & \pm 3.2 & $1566 \pm 26$ & 55 \\
\hline $\mathrm{AD} \quad 905$ & & $1096 \pm 14$ & & $\mathrm{AD}$ & 435 & \pm 3.2 & $1568 \pm 26$ & 15 \\
\hline AD 895 & -1 & $1150 \pm 25$ & 1055 & $\mathrm{AD}$ & 425 & \pm 2.4 & $1623 \pm 20$ & 525 \\
\hline AD 885 & 2.2 & $1175 \pm 18$ & & $\mathrm{AD}$ & 415 & $1 \pm 3.2$ & $1656 \pm 26$ & 535 \\
\hline AD 875 & -1 & $1188 \pm 24$ & & $\mathrm{AD}$ & 405 & \pm 2.9 & $1679 \pm 24$ & 545 \\
\hline $\mathrm{AD} \quad 865$ & -1 & $1207 \pm 16$ & & $A D$ & 395 & \pm 2.4 & $1679 \pm 20$ & 555 \\
\hline $\mathrm{AD} \quad 855$ & -1 & $1222 \pm 18$ & & $\mathrm{AD}$ & 385 & \pm 2.2 & $1692 \pm 18$ & 65 \\
\hline AD 845 & & $1206 \pm 16$ & & $\mathrm{AD}$ & 375 & \pm 1.8 & $1723 \pm 15$ & 75 \\
\hline $\mathrm{AD} \quad 835$ & -1 & $1196 \pm 23$ & & $\mathrm{AD}$ & 365 & \pm 2.9 & $1688 \pm 24$ & 585 \\
\hline AD 825 & -14 & $1206 \pm 16$ & & $\mathrm{AD}$ & 355 & \pm 3.2 & $1717 \pm 26$ & 95 \\
\hline $\mathrm{AD} \quad 815$ & -11 & $1198 \pm 24$ & & $\mathrm{AD}$ & 345 & $6 \pm 3.0$ & $1711 \pm 24$ & 605 \\
\hline AD 805 & -12 & $1212 \pm 17$ & & $\mathrm{AD}$ & 335 & $7 \pm 3.2$ & $1738 \pm 26$ & 1615 \\
\hline AD 795 & 1.7 & $1246 \pm 14$ & & $\mathrm{AD}$ & 325 & \pm 3.2 & $1736 \pm 27$ & 625 \\
\hline AD 785 & & $1181 \pm 13$ & & $\mathrm{AD}$ & 315 & \pm 3.2 & $1792 \pm 26$ & 35 \\
\hline $\mathrm{AD} \quad 775$ & & $1261 \pm 18$ & & $\mathrm{AD}$ & 305 & \pm 3.1 & $1790 \pm 25$ & 1645 \\
\hline AD 765 & -1 & $1291 \pm 14$ & & $\mathrm{AD}$ & 295 & $1 \pm 3.0$ & $1740 \pm 24$ & 1655 \\
\hline $\mathrm{AD} \quad 755$ & & $1310 \pm 12$ & & $\mathrm{AD}$ & 285 & $8 \pm 3.1$ & $1779 \pm 25$ & 665 \\
\hline AD 745 & $-12.8 \pm 1.8$ & $1275 \pm 14$ & 1205 & $\mathrm{AD}$ & 275 & $-12.2 \pm 3.0$ & $1726 \pm 24$ & 1675 \\
\hline $\mathrm{AD} \quad 735$ & \pm 1.6 & $1264 \pm 13$ & 1215 & $\mathrm{AD}$ & 265 & $7 \pm 2.3$ & $1716 \pm 18$ & 1685 \\
\hline AD 725 & & $1278 \pm 14$ & 1225 & $\mathrm{AD}$ & 255 & $-17.2 \pm 1.7$ & $1787 \pm 14$ & 1695 \\
\hline $\mathrm{AD} \quad 715$ & & $1279 \pm 17$ & 123 & $\mathrm{AD}$ & 245 & $8 \pm 1.7$ & $1785 \pm 13$ & 1705 \\
\hline $\mathrm{AD} \quad 705$ & & $1299 \pm 17$ & & $\mathrm{AD}$ & 235 & $-19.0 \pm 2.3$ & $1821 \pm 19$ & 1715 \\
\hline AD 695 & & $1270 \pm 26$ & & $\mathrm{AD}$ & 225 & $-18.7 \pm 3.2$ & $1828 \pm 26$ & 1725 \\
\hline AD 685 & $-14.2 \pm 3.5$ & $1345 \pm 28$ & 1265 & $\mathrm{AD}$ & 215 & $-20.3 \pm 3.2$ & $1851 \pm 27$ & 1735 \\
\hline $\mathrm{AD} \quad 675$ & $-12.5 \pm 3.3$ & $1340 \pm 27$ & 1275 & $\mathrm{AD}$ & 205 & $-20.1 \pm 2.3$ & $1860 \pm 19$ & 1745 \\
\hline $\mathrm{AD} \quad 665$ & $-12.5 \pm 3.3$ & $1350 \pm 27$ & 1285 & $\mathrm{AD}$ & 195 & $-16.2 \pm 3.2$ & $1837 \pm 26$ & 1755 \\
\hline
\end{tabular}


TABLE 1. (Continued)

\begin{tabular}{|c|c|c|c|c|c|c|c|}
\hline \multirow[b]{2}{*}{ Cal $\mathrm{AD} / \mathrm{BC}$} & \multicolumn{3}{|c|}{${ }^{14} \mathrm{C}$} & \multirow[b]{2}{*}{$\mathrm{Cal} \mathrm{AD} / \mathrm{BC}$} & \multicolumn{3}{|c|}{${ }^{14} \mathrm{C}$} \\
\hline & $\Delta^{14} \mathrm{C} \% 0$ & age (BP) & Cal BP & & $\Delta{ }^{14} \mathrm{C} \% 0$ & age (BP) & Cal BP \\
\hline $\mathrm{AD} \quad 185$ & $-13.0 \pm 2.9$ & $1821 \pm 24$ & 1765 & 285 BC & $-10.5 \pm 2.9$ & $2256 \pm 24$ & 2234 \\
\hline AD 175 & $-15.1 \pm 3.2$ & $1848 \pm 26$ & 1775 & $295 \mathrm{BC}$ & $-5.5 \pm 2.2$ & $2225 \pm 18$ & 2244 \\
\hline AD 165 & $-14.2 \pm 3.5$ & $1850 \pm 28$ & 1785 & $305 \mathrm{BC}$ & $-2.5 \pm 3.1$ & $2211 \pm 25$ & 2254 \\
\hline AD 155 & $-13.6 \pm 3.0$ & $1854 \pm 24$ & 1795 & $315 \mathrm{BC}$ & $-1.1 \pm 3.1$ & $2210 \pm 25$ & 2264 \\
\hline AD 145 & $-12.3 \pm 3.2$ & $1854 \pm 26$ & 1805 & $325 \mathrm{BC}$ & $3.2 \pm 2.2$ & $2185 \pm 18$ & 2274 \\
\hline AD 135 & $-10.2 \pm 2.1$ & $1847 \pm 17$ & 1815 & 335 вC & $4.9 \pm 3.1$ & $2181 \pm 25$ & 2284 \\
\hline AD 125 & $-16.9 \pm 1.7$ & $1911 \pm 14$ & 1825 & 345 BC & $5.0 \pm 2.2$ & $2189 \pm 18$ & 2294 \\
\hline AD 115 & $-15.9 \pm 3.5$ & $1913 \pm 28$ & 1835 & $355 \mathrm{BC}$ & $-0.8 \pm 2.2$ & $2246 \pm 18$ & 2304 \\
\hline AD 105 & $-15.5 \pm 3.3$ & $1919 \pm 27$ & 1845 & $365 \mathrm{BC}$ & $-4.1 \pm 2.2$ & $2282 \pm 18$ & 2314 \\
\hline AD 95 & $-10.6 \pm 1.9$ & $1888 \pm 16$ & 1855 & $375 \mathrm{BC}$ & $-2.3 \pm 2.2$ & $2278 \pm 18$ & 2324 \\
\hline AD 85 & $-11.5 \pm 3.2$ & $1906 \pm 26$ & 1865 & $385 \mathrm{BC}$ & $-3.3 \pm 3.1$ & $2295 \pm 25$ & 2334 \\
\hline AD 75 & $-13.3 \pm 2.2$ & $1930 \pm 18$ & 1875 & 395 вС & $-7.8 \pm 2.3$ & $2341 \pm 19$ & 2344 \\
\hline $\mathrm{AD}$ & $-14.2 \pm 2.2$ & $1947 \pm 18$ & 1885 & $405 \mathrm{BC}$ & $-12.6 \pm 1.8$ & $2390 \pm 14$ & 2354 \\
\hline $\mathrm{AD}$ & $-15.2 \pm 1.9$ & $1965 \pm 15$ & 1895 & 415 вC & $-18.7 \pm 3.2$ & $2449 \pm 26$ & 2364 \\
\hline $\mathrm{AD}$ & $-18.3 \pm 1.6$ & $2000 \pm 13$ & 1905 & $425 \mathrm{BC}$ & $-16.0 \pm 3.3$ & $2437 \pm 27$ & 2374 \\
\hline $\mathrm{AD}$ & $-11.0 \pm 2.5$ & $1950 \pm 20$ & 1915 & $435 \mathrm{BC}$ & $-18.9 \pm 3.1$ & $2471 \pm 25$ & 2384 \\
\hline $\mathrm{AD}$ & $-15.3 \pm 2.2$ & $1995 \pm 18$ & 1925 & 445 BC & $-17.8 \pm 5.3$ & $2471 \pm 44$ & 2394 \\
\hline $\mathrm{AD}$ & $-15.9 \pm 1.8$ & $2010 \pm 15$ & 1935 & $455 \mathrm{BC}$ & $-21.0 \pm 3.6$ & $2507 \pm 30$ & 2404 \\
\hline $\mathrm{AD}$ & $-12.6 \pm 1.6$ & $1993 \pm 13$ & 1945 & $465 \mathrm{BC}$ & $-13.3 \pm 4.0$ & $2454 \pm 33$ & 2414 \\
\hline $5 \mathrm{BC}$ & $-20.2 \pm 1.8$ & $2063 \pm 15$ & 1954 & 475 BC & $-7.4 \pm 2.3$ & $2416 \pm 19$ & 2424 \\
\hline $15 \mathrm{BC}$ & $-12.9 \pm 1.6$ & $2012 \pm 13$ & 1964 & $485 \mathrm{BC}$ & $-10.9 \pm 3.3$ & $2454 \pm 26$ & 2434 \\
\hline $25 \mathrm{BC}$ & $-15.6 \pm 2.1$ & $2044 \pm 17$ & 1974 & $495 \mathrm{BC}$ & $-7.3 \pm 2.5$ & $2434 \pm 20$ & 2444 \\
\hline $35 \mathrm{BC}$ & $-11.2 \pm 1.2$ & $2018 \pm 10$ & 1984 & $505 \mathrm{BC}$ & $-8.0 \pm 3.3$ & $2450 \pm 26$ & 2454 \\
\hline $45 \mathrm{BC}$ & $-14.3 \pm 1.7$ & $2053 \pm 14$ & 1994 & 515 вC & $-6.1 \pm 2.5$ & $2444 \pm 20$ & 2464 \\
\hline $55 \mathrm{BC}$ & $-17.3 \pm 1.5$ & $2087 \pm 12$ & 2004 & $525 \mathrm{BC}$ & $-10.1 \pm 2.7$ & $2487 \pm 22$ & 2474 \\
\hline $65 \mathrm{BC}$ & $-18.0 \pm 1.8$ & $2103 \pm 15$ & 2014 & $535 \mathrm{BC}$ & $-6.8 \pm 1.8$ & $2469 \pm 14$ & 2484 \\
\hline $75 \mathrm{BC}$ & $-15.6 \pm 2.3$ & $2094 \pm 19$ & 2024 & 545 вс & $-8.6 \pm 1.7$ & $2493 \pm 13$ & 2494 \\
\hline $85 \mathrm{BC}$ & $-12.4 \pm 1.8$ & $2076 \pm 15$ & 2034 & $555 \mathrm{BC}$ & $-7.1 \pm 2.9$ & $2491 \pm 23$ & 2504 \\
\hline $95 \mathrm{BC}$ & $-15.1 \pm 2.2$ & $2108 \pm 18$ & 2044 & $565 \mathrm{BC}$ & $-7.0 \pm 3.6$ & $2500 \pm 29$ & 2514 \\
\hline $105 \mathrm{BC}$ & $-12.3 \pm 2.0$ & $2095 \pm 16$ & 2054 & 575 вC & $-6.7 \pm 3.5$ & $2508 \pm 28$ & 2524 \\
\hline $115 \mathrm{BC}$ & $-13.0 \pm 1.9$ & $2111 \pm 16$ & 2064 & 585 BC & $-0.4 \pm 3.5$ & $2466 \pm 29$ & 2534 \\
\hline $125 \mathrm{BC}$ & $-14.2 \pm 1.9$ & $2130 \pm 16$ & 2074 & 595 вC & $-7.2 \pm 2.8$ & $2530 \pm 23$ & 2544 \\
\hline $135 \mathrm{BC}$ & $-10.7 \pm 2.3$ & $2111 \pm 19$ & 2084 & $605 \mathrm{BC}$ & $-5.1 \pm 3.0$ & $2524 \pm 24$ & 2554 \\
\hline $145 \mathrm{BC}$ & $-8.5 \pm 1.9$ & $2103 \pm 16$ & 2094 & $615 \mathrm{BC}$ & $-2.0 \pm 2.6$ & $2508 \pm 21$ & 2564 \\
\hline 155 BC & $-6.7 \pm 3.4$ & $2099 \pm 27$ & 2104 & $625 \mathrm{BC}$ & $-1.9 \pm 2.7$ & $2517 \pm 22$ & 2574 \\
\hline $165 \mathrm{BC}$ & $-7.7 \pm 2.2$ & $2117 \pm 18$ & 2114 & $635 \mathrm{BC}$ & $2.6 \pm 2.3$ & $2488 \pm 19$ & 2584 \\
\hline $175 \mathrm{BC}$ & $-14.2 \pm 3.3$ & $2179 \pm 27$ & 2124 & 645 BC & $3.6 \pm 2.3$ & $2492 \pm 19$ & 2594 \\
\hline 185 вC & $-12.6 \pm 3.3$ & $2176 \pm 27$ & 2134 & $655 \mathrm{BC}$ & $4.9 \pm 2.1$ & $2492 \pm 17$ & 2604 \\
\hline $195 \mathrm{BC}$ & $-8.0 \pm 2.2$ & $2149 \pm 18$ & 2144 & $661 \mathrm{BC}$ & $11.6 \pm 3.2$ & $2444 \pm 25$ & 2610 \\
\hline $205 \mathrm{BC}$ & $-12.6 \pm 3.0$ & $2195 \pm 25$ & 2154 & $696 \mathrm{BC}$ & $12.7 \pm 5.3$ & $2469 \pm 42$ & 2645 \\
\hline $215 \mathrm{BC}$ & $-17.4 \pm 1.7$ & $2244 \pm 14$ & 2164 & 705 вC & $13.5 \pm 5.4$ & $2473 \pm 43$ & 2654 \\
\hline $225 \mathrm{BC}$ & $-9.7 \pm 2.4$ & $2192 \pm 20$ & 2174 & 715 BC & $14.0 \pm 5.4$ & $2479 \pm 43$ & 2664 \\
\hline $235 \mathrm{BC}$ & $-16.6 \pm 1.8$ & $2258 \pm 15$ & 2184 & $731 \mathrm{BC}$ & $18.1 \pm 2.3$ & $2461 \pm 18$ & 2680 \\
\hline 245 BC & $-14.1 \pm 2.4$ & $2247 \pm 19$ & 2194 & $751 \mathrm{BC}$ & $20.5 \pm 3.4$ & $2461 \pm 27$ & 2700 \\
\hline $255 \mathrm{BC}$ & $-11.6 \pm 3.2$ & $2236 \pm 26$ & 2204 & $771 \mathrm{BC}$ & $9.9 \pm 3.2$ & $2565 \pm 26$ & 2720 \\
\hline $265 \mathrm{BC}$ & $-15.8 \pm 3.1$ & $2280 \pm 25$ & 2214 & 785 вC & $11.9 \pm 2.5$ & $2563 \pm 20$ & 2734 \\
\hline $275 \mathrm{BC}$ & $-9.8 \pm 2.2$ & $2241 \pm 18$ & 2224 & 795 BC & $5.7 \pm 2.5$ & $2622 \pm 20$ & 2744 \\
\hline
\end{tabular}


TABLE 1. (Continued)

\begin{tabular}{|c|c|c|c|c|c|c|c|}
\hline \multirow[b]{2}{*}{ Cal $\mathrm{AD} / \mathrm{BC}$} & \multicolumn{3}{|c|}{${ }^{14} \mathrm{C}$} & \multirow[b]{2}{*}{ Cal AD/BC } & \multicolumn{3}{|c|}{${ }^{14} \mathrm{C}$} \\
\hline & $\Delta^{14} \mathrm{C} \% 0$ & age (BP) & Cal BP & & $\Delta^{14} \mathrm{C} \% 0$ & age (BP) & Cal BP \\
\hline $805 \mathrm{BC}$ & $3.0 \pm 2.5$ & $2654 \pm 20$ & 2754 & $1275 \mathrm{BC}$ & $10.2 \pm 3.4$ & $3053 \pm 27$ & 3224 \\
\hline $815 \mathrm{BC}$ & $-0.3 \pm 3.3$ & $2690 \pm 27$ & 2764 & $1285 \mathrm{BC}$ & $16.4 \pm 3.6$ & $3013 \pm 28$ & 3234 \\
\hline $825 \mathrm{BC}$ & $1.1 \pm 2.6$ & $2688 \pm 21$ & 2774 & $1295 \mathrm{BC}$ & $12.5 \pm 3.5$ & $3054 \pm 28$ & 3244 \\
\hline $835 \mathrm{BC}$ & $-5.7 \pm 2.5$ & $2752 \pm 20$ & 2784 & $1305 \mathrm{BC}$ & $14.6 \pm 3.7$ & $3047 \pm 29$ & 3254 \\
\hline $845 \mathrm{BC}$ & $-5.8 \pm 2.5$ & $2763 \pm 20$ & 2794 & $1315 \mathrm{BC}$ & $14.3 \pm 3.5$ & $3059 \pm 28$ & 3264 \\
\hline $855 \mathrm{BC}$ & $-4.1 \pm 3.3$ & $2759 \pm 27$ & 2804 & $1325 \mathrm{BC}$ & $8.2 \pm 3.0$ & $3117 \pm 24$ & 3274 \\
\hline $865 \mathrm{BC}$ & $-2.3 \pm 3.2$ & $2755 \pm 26$ & 2814 & $1335 \mathrm{BC}$ & $15.3 \pm 3.5$ & $3070 \pm 27$ & 3284 \\
\hline $875 \mathrm{BC}$ & $-1.4 \pm 3.4$ & $2757 \pm 27$ & 2824 & $1345 \mathrm{BC}$ & $17.5 \pm 3.6$ & $3063 \pm 28$ & 3294 \\
\hline $885 \mathrm{BC}$ & $3.6 \pm 3.4$ & $2727 \pm 27$ & 2834 & $1355 \mathrm{BC}$ & $15.3 \pm 3.5$ & $3090 \pm 27$ & 3304 \\
\hline $895 \mathrm{BC}$ & $1.7 \pm 3.3$ & $2752 \pm 27$ & 2844 & $1365 \mathrm{BC}$ & $22.6 \pm 3.3$ & $3042 \pm 26$ & 3314 \\
\hline $905 \mathrm{BC}$ & $-2.3 \pm 3.2$ & $2793 \pm 26$ & 2854 & $1375 \mathrm{BC}$ & $16.6 \pm 3.2$ & $3100 \pm 25$ & 3324 \\
\hline $915 \mathrm{BC}$ & $-2.0 \pm 3.3$ & $2801 \pm 27$ & 2864 & $1385 \mathrm{BC}$ & $17.3 \pm 3.3$ & $3104 \pm 26$ & 3334 \\
\hline $925 \mathrm{BC}$ & $-1.9 \pm 3.3$ & $2810 \pm 27$ & 2874 & $1395 \mathrm{BC}$ & $16.4 \pm 2.4$ & $3121 \pm 19$ & 3344 \\
\hline $935 \mathrm{BC}$ & $-0.9 \pm 2.5$ & $2811 \pm 20$ & 2884 & $1405 \mathrm{BC}$ & $18.1 \pm 3.3$ & $3117 \pm 26$ & 3354 \\
\hline $945 \mathrm{BC}$ & $-3.2 \pm 2.4$ & $2840 \pm 19$ & 2894 & $1415 \mathrm{BC}$ & $10.8 \pm 3.3$ & $3184 \pm 26$ & 3364 \\
\hline $955 \mathrm{BC}$ & $-2.0 \pm 2.6$ & $2839 \pm 21$ & 2904 & $1425 \mathrm{BC}$ & $18.8 \pm 3.4$ & $3131 \pm 27$ & 3374 \\
\hline 965 BC & $7.7 \pm 3.4$ & $2772 \pm 27$ & 2914 & $1435 \mathrm{BC}$ & $11.8 \pm 2.4$ & $3196 \pm 19$ & 3384 \\
\hline $975 \mathrm{BC}$ & $-1.6 \pm 2.4$ & $2856 \pm 19$ & 2924 & $1445 \mathrm{BC}$ & $13.8 \pm 2.4$ & $3189 \pm 19$ & 3394 \\
\hline $985 \mathrm{BC}$ & $0.4 \pm 3.2$ & $2849 \pm 25$ & 2934 & $1455 \mathrm{BC}$ & $11.6 \pm 2.3$ & $3216 \pm 18$ & 3404 \\
\hline 995 BC & $3.6 \pm 3.2$ & $2833 \pm 25$ & 2944 & $1465 \mathrm{BC}$ & $9.9 \pm 2.4$ & $3240 \pm 19$ & 3414 \\
\hline $1005 \mathrm{BC}$ & $3.2 \pm 3.3$ & $2847 \pm 27$ & 2954 & $1475 \mathrm{BC}$ & $12.9 \pm 2.4$ & $3226 \pm 19$ & 3424 \\
\hline $1015 \mathrm{BC}$ & $-0.6 \pm 3.2$ & $2887 \pm 26$ & 2964 & $1485 \mathrm{BC}$ & $17.2 \pm 2.6$ & $3202 \pm 21$ & 3434 \\
\hline $1025 \mathrm{BC}$ & $-2.2 \pm 3.5$ & $2909 \pm 28$ & 2974 & $1495 \mathrm{BC}$ & $12.8 \pm 3.7$ & $3246 \pm 29$ & 3444 \\
\hline $1035 \mathrm{BC}$ & $0.3 \pm 3.4$ & $2899 \pm 27$ & 2984 & $1505 \mathrm{BC}$ & $11.8 \pm 3.3$ & $3264 \pm 26$ & 3454 \\
\hline $1045 \mathrm{BC}$ & $6.2 \pm 3.5$ & $2861 \pm 28$ & 2994 & $1515 \mathrm{BC}$ & $12.0 \pm 3.5$ & $3272 \pm 28$ & 3464 \\
\hline $1055 \mathrm{BC}$ & $-2.6 \pm 3.2$ & $2941 \pm 26$ & 3004 & $1525 \mathrm{BC}$ & $11.1 \pm 3.2$ & $3289 \pm 25$ & 3474 \\
\hline $1065 \mathrm{BC}$ & $4.4 \pm 3.6$ & $2895 \pm 29$ & 3014 & $1535 \mathrm{BC}$ & $7.2 \pm 3.4$ & $3329 \pm 27$ & 3484 \\
\hline $1075 \mathrm{BC}$ & $4.0 \pm 3.5$ & $2908 \pm 28$ & 3024 & $1545 \mathrm{BC}$ & $10.0 \pm 3.5$ & $3316 \pm 28$ & 3494 \\
\hline $1085 \mathrm{BC}$ & $1.4 \pm 3.6$ & $2939 \pm 29$ & 3034 & $1555 \mathrm{BC}$ & $10.8 \pm 3.3$ & $3320 \pm 27$ & 3504 \\
\hline $1095 \mathrm{BC}$ & $4.1 \pm 2.3$ & $2926 \pm 19$ & 3044 & $1565 \mathrm{BC}$ & $8.8 \pm 5.8$ & $3346 \pm 46$ & 3514 \\
\hline 1105 вC & $5.3 \pm 2.5$ & $2927 \pm 20$ & 3054 & $1575 \mathrm{BC}$ & $13.2 \pm 5.8$ & $3320 \pm 46$ & 3524 \\
\hline $1115 \mathrm{BC}$ & $0.4 \pm 3.8$ & $2976 \pm 30$ & 3064 & $1585 \mathrm{BC}$ & $14.2 \pm 3.6$ & $3323 \pm 28$ & 3534 \\
\hline $1125 \mathrm{BC}$ & $7.5 \pm 3.4$ & $2928 \pm 27$ & 3074 & $1595 \mathrm{BC}$ & $20.1 \pm 2.8$ & $3286 \pm 22$ & 3544 \\
\hline $1135 \mathrm{BC}$ & $-2.3 \pm 2.3$ & $3017 \pm 18$ & 3084 & $1605 \mathrm{BC}$ & $14.5 \pm 2.6$ & $3340 \pm 20$ & 3554 \\
\hline 1145 ВС & $10.3 \pm 3.4$ & $2926 \pm 27$ & 3094 & $1615 \mathrm{BC}$ & $15.1 \pm 2.8$ & $3345 \pm 22$ & 3564 \\
\hline $1155 \mathrm{BC}$ & $7.4 \pm 3.5$ & $2959 \pm 28$ & 3104 & $1625 \mathrm{BC}$ & $13.5 \pm 2.7$ & $3367 \pm 21$ & 3574 \\
\hline $1165 \mathrm{BC}$ & $8.3 \pm 3.3$ & $2961 \pm 26$ & 3114 & $1635 \mathrm{BC}$ & $13.5 \pm 3.6$ & $3376 \pm 28$ & 3584 \\
\hline $1175 \mathrm{BC}$ & $8.4 \pm 4.2$ & $2970 \pm 33$ & 3124 & $1645 \mathrm{BC}$ & $11.3 \pm 2.0$ & $3404 \pm 16$ & 3594 \\
\hline $1185 \mathrm{BC}$ & $16.0 \pm 3.5$ & $2919 \pm 28$ & 3134 & $1655 \mathrm{BC}$ & $19.0 \pm 2.6$ & $3353 \pm 21$ & 3604 \\
\hline $1195 \mathrm{BC}$ & $4.9 \pm 3.2$ & $3018 \pm 26$ & 3144 & $1665 \mathrm{BC}$ & $11.5 \pm 3.7$ & $3422 \pm 30$ & 3614 \\
\hline $1205 \mathrm{BC}$ & $16.1 \pm 3.6$ & $2938 \pm 28$ & 3154 & $1675 \mathrm{BC}$ & $24.1 \pm 3.5$ & $3332 \pm 28$ & 3624 \\
\hline $1215 \mathrm{BC}$ & $8.3 \pm 1.9$ & $3009 \pm 15$ & 3164 & $1685 \mathrm{BC}$ & $20.2 \pm 3.5$ & $3372 \pm 28$ & 3634 \\
\hline $1225 \mathrm{BC}$ & $8.5 \pm 3.3$ & $3018 \pm 27$ & 3174 & $1695 \mathrm{BC}$ & $11.2 \pm 2.4$ & $3453 \pm 20$ & 3644 \\
\hline $1235 \mathrm{BC}$ & $12.9 \pm 3.6$ & $2993 \pm 29$ & 3184 & $1705 \mathrm{BC}$ & $13.1 \pm 3.6$ & $3448 \pm 28$ & 3654 \\
\hline $1245 \mathrm{BC}$ & $11.9 \pm 3.5$ & $3011 \pm 28$ & 3194 & $1715 \mathrm{BC}$ & $19.0 \pm 3.5$ & $3410 \pm 28$ & 3664 \\
\hline $1255 \mathrm{BC}$ & $14.5 \pm 2.7$ & $3007 \pm 22$ & 3204 & $1725 \mathrm{BC}$ & $19.1 \pm 3.5$ & $3420 \pm 28$ & 3674 \\
\hline $1265 \mathrm{BC}$ & $8.0 \pm 3.5$ & $3061 \pm 28$ & 3214 & $1735 \mathrm{BC}$ & $17.3 \pm 3.5$ & $3443 \pm 28$ & 3684 \\
\hline
\end{tabular}


TABLE 1. (Continued)

\begin{tabular}{|c|c|c|c|c|c|c|c|}
\hline \multirow[b]{2}{*}{ Cal AD/BC } & \multicolumn{3}{|c|}{${ }^{14} \mathrm{C}$} & \multirow[b]{2}{*}{$\mathrm{Cal} \mathrm{AD} / \mathrm{BC}$} & \multicolumn{3}{|c|}{${ }^{14} \mathrm{C}$} \\
\hline & $\Delta^{14} \mathrm{C} \% 0$ & age (BP) & Cal BP & & $\Delta^{14} \mathrm{C} \% 0$ & age (BP) & Cal BP \\
\hline $1745 \mathrm{BC}$ & $12.6 \pm 3.6$ & $3491 \pm 28$ & 3694 & $2215 \mathrm{BC}$ & $27.2 \pm 2.5$ & $3832 \pm 20$ & 4164 \\
\hline $1755 \mathrm{BC}$ & $12.9 \pm 2.5$ & $3498 \pm 20$ & 3704 & 2225 BC & $32.1 \pm 2.1$ & $3804 \pm 17$ & 4174 \\
\hline 1765 вC & $17.9 \pm 2.8$ & $3468 \pm 22$ & 3714 & $2235 \mathrm{BC}$ & $26.6 \pm 3.9$ & $3857 \pm 31$ & 4184 \\
\hline $1775 \mathrm{BC}$ & $13.2 \pm 2.7$ & $3515 \pm 21$ & 3724 & 2245 BC & $29.2 \pm 2.6$ & $3846 \pm 21$ & 4194 \\
\hline 1785 вС & $12.4 \pm 2.4$ & $3531 \pm 19$ & 3734 & 2255 вС & $34.9 \pm 2.5$ & $3811 \pm 19$ & 4204 \\
\hline $1795 \mathrm{BC}$ & $15.2 \pm 2.4$ & $3518 \pm 19$ & 3744 & 2265 вС & $37.0 \pm 3.6$ & $3805 \pm 28$ & 4214 \\
\hline $1805 \mathrm{BC}$ & $19.3 \pm 2.4$ & $3495 \pm 19$ & 3754 & 2275 вС & $34.6 \pm 4.3$ & $3833 \pm 34$ & 4224 \\
\hline 1815 BC & $17.0 \pm 2.5$ & $3524 \pm 20$ & 3764 & 2285 BC & $34.4 \pm 3.0$ & $3844 \pm 23$ & 4234 \\
\hline $1825 \mathrm{BC}$ & $19.7 \pm 3.6$ & $3512 \pm 28$ & 3774 & $2295 \mathrm{BC}$ & $32.2 \pm 2.7$ & $3871 \pm 21$ & 4244 \\
\hline $1835 \mathrm{BC}$ & $13.6 \pm 3.4$ & $3570 \pm 26$ & 3784 & 2305 BC & $36.5 \pm 4.0$ & $3847 \pm 31$ & 4254 \\
\hline $1845 \mathrm{BC}$ & $22.6 \pm 3.6$ & $3509 \pm 28$ & 3794 & $2315 \mathrm{BC}$ & $33.8 \pm 3.6$ & $3878 \pm 28$ & 4264 \\
\hline $1855 \mathrm{BC}$ & $25.7 \pm 3.6$ & $3494 \pm 28$ & 3804 & $2325 \mathrm{BC}$ & $36.8 \pm 2.6$ & $3864 \pm 20$ & 4274 \\
\hline $1865 \mathrm{BC}$ & $23.3 \pm 3.6$ & $3523 \pm 28$ & 3814 & 2335 вC & $35.9 \pm 3.6$ & $3881 \pm 28$ & 4284 \\
\hline $1875 \mathrm{BC}$ & $24.7 \pm 2.5$ & $3522 \pm 19$ & 3824 & $2345 \mathrm{BC}$ & $35.0 \pm 2.6$ & $3898 \pm 20$ & 4294 \\
\hline $1885 \mathrm{BC}$ & $19.2 \pm 3.4$ & $3575 \pm 27$ & 3834 & $2355 \mathrm{BC}$ & $34.6 \pm 3.7$ & $3911 \pm 29$ & 4304 \\
\hline 1895 вС & $18.1 \pm 3.6$ & $3593 \pm 28$ & 3844 & 2365 BC & $34.5 \pm 2.6$ & $3921 \pm 20$ & 4314 \\
\hline $1905 \mathrm{BC}$ & $22.4 \pm 3.5$ & $3569 \pm 28$ & 3854 & 2375 вC & $37.2 \pm 3.6$ & $3910 \pm 28$ & 4324 \\
\hline 1915 BC & $23.4 \pm 3.5$ & $3570 \pm 28$ & 3864 & $2385 \mathrm{BC}$ & $42.8 \pm 2.6$ & $3876 \pm 20$ & 4334 \\
\hline 1925 вC & $19.9 \pm 2.7$ & $3608 \pm 22$ & 3874 & 2395 вC & $47.7 \pm 4.1$ & $3848 \pm 31$ & 4344 \\
\hline 1935 BC & $21.0 \pm 3.2$ & $3609 \pm 25$ & 3884 & $2405 \mathrm{BC}$ & $40.5 \pm 2.7$ & $3914 \pm 21$ & 4354 \\
\hline 1945 BC & $20.3 \pm 3.7$ & $3624 \pm 29$ & 3894 & $2415 \mathrm{BC}$ & $39.6 \pm 2.9$ & $3930 \pm 22$ & 4364 \\
\hline $1955 \mathrm{BC}$ & $20.3 \pm 3.6$ & $3633 \pm 28$ & 3904 & 2425 BC & $44.2 \pm 3.6$ & $3904 \pm 28$ & 4374 \\
\hline 1965 вC & $21.7 \pm 3.5$ & $3633 \pm 28$ & 3914 & $2435 \mathrm{BC}$ & $43.4 \pm 1.9$ & $3920 \pm 15$ & 4384 \\
\hline 1975 вC & $20.5 \pm 3.5$ & $3651 \pm 27$ & 3924 & $2445 \mathrm{BC}$ & $45.3 \pm 3.8$ & $3915 \pm 29$ & 4394 \\
\hline 1985 вС & $16.6 \pm 3.5$ & $3692 \pm 28$ & 3934 & 2465 BC & $44.8 \pm 3.7$ & $3939 \pm 28$ & 4414 \\
\hline 1995 BC & $25.9 \pm 3.5$ & $3628 \pm 27$ & 3944 & $2475 \mathrm{BC}$ & $34.4 \pm 2.6$ & $4029 \pm 20$ & 4424 \\
\hline $2005 \mathrm{BC}$ & $29.2 \pm 3.6$ & $3613 \pm 28$ & 3954 & 2485 BC & $38.1 \pm 2.7$ & $4010 \pm 21$ & 4434 \\
\hline 2015 BC & $23.5 \pm 3.4$ & $3667 \pm 27$ & 3964 & 2495 вC & $32.7 \pm 3.9$ & $4062 \pm 30$ & 4444 \\
\hline 2025 BC & $25.9 \pm 3.5$ & $3658 \pm 28$ & 3974 & $2505 \mathrm{BC}$ & $30.5 \pm 2.9$ & $4088 \pm 23$ & 4454 \\
\hline $2035 \mathrm{BC}$ & $22.2 \pm 2.2$ & $3697 \pm 17$ & 3984 & $2515 \mathrm{BC}$ & $38.2 \pm 2.9$ & $4038 \pm 22$ & 4464 \\
\hline 2045 BC & $20.1 \pm 2.5$ & $3723 \pm 19$ & 3994 & $2525 \mathrm{BC}$ & $43.0 \pm 2.0$ & $4011 \pm 16$ & 4474 \\
\hline $2055 \mathrm{BC}$ & $20.1 \pm 2.5$ & $3732 \pm 20$ & 4004 & $2535 \mathrm{BC}$ & $45.7 \pm 2.7$ & $4000 \pm 21$ & 4484 \\
\hline 2065 BC & $25.5 \pm 3.5$ & $3700 \pm 28$ & 4014 & $2545 \mathrm{BC}$ & $45.9 \pm 2.7$ & $4008 \pm 21$ & 4494 \\
\hline 2075 BC & $18.9 \pm 2.5$ & $3761 \pm 20$ & 4024 & $2555 \mathrm{BC}$ & $46.2 \pm 2.5$ & $4015 \pm 19$ & 4504 \\
\hline 2085 BC & $29.5 \pm 3.6$ & $3688 \pm 28$ & 4034 & $2565 \mathrm{BC}$ & $44.8 \pm 2.5$ & $4036 \pm 19$ & 4514 \\
\hline 2095 BC & $27.9 \pm 3.5$ & $3710 \pm 27$ & 4044 & $2575 \mathrm{BC}$ & $40.8 \pm 3.0$ & $4077 \pm 23$ & 4524 \\
\hline $2105 \mathrm{BC}$ & $32.6 \pm 2.5$ & $3684 \pm 19$ & 4054 & $2585 \mathrm{BC}$ & $38.1 \pm 2.4$ & $4107 \pm 19$ & 4534 \\
\hline $2115 \mathrm{BC}$ & $29.9 \pm 3.5$ & $3714 \pm 27$ & 4064 & $2595 \mathrm{BC}$ & $44.3 \pm 2.7$ & $4069 \pm 21$ & 4544 \\
\hline $2125 \mathrm{BC}$ & $32.2 \pm 3.5$ & $3706 \pm 28$ & 4074 & 2605 BC & $37.2 \pm 4.1$ & $4133 \pm 32$ & 4554 \\
\hline 2135 вС & $29.9 \pm 3.5$ & $3733 \pm 27$ & 4084 & 2615 BC & $47.1 \pm 2.7$ & $4067 \pm 21$ & 4564 \\
\hline 2145 BC & $25.1 \pm 2.2$ & $3781 \pm 17$ & 4094 & $2625 \mathrm{BC}$ & $43.1 \pm 2.8$ & $4108 \pm 22$ & 4574 \\
\hline $2155 \mathrm{BC}$ & $27.3 \pm 2.3$ & $3773 \pm 18$ & 4104 & $2635 \mathrm{BC}$ & $42.0 \pm 2.4$ & $4126 \pm 18$ & 4584 \\
\hline 2165 BC & $29.6 \pm 3.6$ & $3765 \pm 28$ & 4114 & $2645 \mathrm{BC}$ & $36.0 \pm 4.0$ & $4182 \pm 31$ & 4594 \\
\hline 2175 вс & $33.0 \pm 2.4$ & $3748 \pm 18$ & 4124 & $2655 \mathrm{BC}$ & $47.5 \pm 4.0$ & $4101 \pm 31$ & 4604 \\
\hline 2185 BC & $34.6 \pm 2.0$ & $3745 \pm 16$ & 4134 & $2665 \mathrm{BC}$ & $47.2 \pm 2.7$ & $4113 \pm 21$ & 4614 \\
\hline 2195 вC & $33.6 \pm 2.0$ & $3763 \pm 16$ & 4144 & 2675 BC & $45.1 \pm 2.7$ & $4139 \pm 21$ & 4624 \\
\hline $2205 \mathrm{BC}$ & $28.1 \pm 3.0$ & $3816 \pm 24$ & 4154 & $2685 \mathrm{BC}$ & $45.9 \pm 2.8$ & $4142 \pm 21$ & 4634 \\
\hline
\end{tabular}


TABLE 1. (Continued)

\begin{tabular}{|c|c|c|c|c|c|c|c|}
\hline \multicolumn{4}{|c|}{${ }^{14} \mathrm{C}$} & \multicolumn{4}{|c|}{${ }^{14} \mathrm{C}$} \\
\hline Cal $\mathrm{AD} / \mathrm{BC}$ & $\Delta^{14} \mathrm{C} \% 0$ & age (BP) & Cal BP & $\mathrm{Cal} \mathrm{AD} / \mathrm{BC}$ & $\Delta^{14} \mathrm{C} \% 0$ & age (BP) & $\mathrm{Cal} \mathrm{BP}$ \\
\hline $2695 \mathrm{BC}$ & $45.0 \pm 4.1$ & $4159 \pm 31$ & 4644 & 3165 BC & $62.3 \pm 2.8$ & $4484 \pm 21$ & 5114 \\
\hline $2705 \mathrm{BC}$ & $44.6 \pm 2.2$ & $4171 \pm 17$ & 4654 & 3175 вC & $56.3 \pm 4.0$ & $4539 \pm 31$ & 5124 \\
\hline $2715 \mathrm{BC}$ & $42.2 \pm 4.1$ & $4200 \pm 32$ & 4664 & 3185 вС & $60.8 \pm 2.9$ & $4515 \pm 22$ & 5134 \\
\hline $2725 \mathrm{BC}$ & $48.7 \pm 4.1$ & $4160 \pm 32$ & 4674 & 3195 вС & $60.2 \pm 2.8$ & $4529 \pm 21$ & 5144 \\
\hline $2735 \mathrm{BC}$ & $47.2 \pm 3.3$ & $4182 \pm 26$ & 4684 & $3205 \mathrm{BC}$ & $59.5 \pm 4.1$ & $4544 \pm 31$ & 5154 \\
\hline $2745 \mathrm{BC}$ & $51.0 \pm 4.1$ & $4162 \pm 31$ & 4694 & $3215 \mathrm{BC}$ & $64.0 \pm 2.5$ & $4520 \pm 19$ & 5164 \\
\hline $2755 \mathrm{BC}$ & $51.4 \pm 4.1$ & $4169 \pm 31$ & 4704 & $3225 \mathrm{BC}$ & $66.5 \pm 4.1$ & $4511 \pm 31$ & 5174 \\
\hline $2765 \mathrm{BC}$ & $44.2 \pm 4.3$ & $4234 \pm 33$ & 4714 & $3235 \mathrm{BC}$ & $71.2 \pm 4.1$ & $4485 \pm 31$ & 5184 \\
\hline $2775 \mathrm{BC}$ & $51.8 \pm 3.5$ & $4185 \pm 27$ & 4724 & $3245 \mathrm{BC}$ & $77.1 \pm 4.2$ & $4451 \pm 31$ & 5194 \\
\hline $2785 \mathrm{BC}$ & $54.6 \pm 2.8$ & $4174 \pm 21$ & 4734 & $3255 \mathrm{BC}$ & $75.3 \pm 4.1$ & $4474 \pm 30$ & 5204 \\
\hline $2795 \mathrm{BC}$ & $52.8 \pm 2.8$ & $4197 \pm 22$ & 4744 & $3265 \mathrm{BC}$ & $73.1 \pm 3.6$ & $4500 \pm 27$ & 5214 \\
\hline $2805 \mathrm{BC}$ & $51.2 \pm 3.8$ & $4219 \pm 29$ & 4754 & $3275 \mathrm{BC}$ & $73.9 \pm 4.4$ & $4504 \pm 33$ & 5224 \\
\hline $2815 \mathrm{BC}$ & $63.5 \pm 2.9$ & $4135 \pm 22$ & 4764 & $3285 \mathrm{BC}$ & $77.8 \pm 4.1$ & $4485 \pm 31$ & 5234 \\
\hline $2825 \mathrm{BC}$ & $69.6 \pm 4.3$ & $4099 \pm 33$ & 4774 & 3295 BC & $79.2 \pm 4.1$ & $4484 \pm 31$ & 5244 \\
\hline $2835 \mathrm{BC}$ & $64.4 \pm 3.9$ & $4147 \pm 30$ & 4784 & 3305 BC & $78.8 \pm 4.2$ & $4496 \pm 31$ & 5254 \\
\hline $2845 \mathrm{BC}$ & $62.6 \pm 3.8$ & $4171 \pm 29$ & 4794 & $3315 \mathrm{BC}$ & $75.4 \pm 3.0$ & $4532 \pm 22$ & 5264 \\
\hline 2855 вС & $63.5 \pm 4.0$ & $4174 \pm 30$ & 4804 & $3325 \mathrm{BC}$ & $81.8 \pm 2.9$ & $4494 \pm 22$ & 5274 \\
\hline 2865 вC & $63.8 \pm 3.7$ & $4181 \pm 28$ & 4814 & $3335 \mathrm{BC}$ & $77.8 \pm 2.6$ & $4533 \pm 20$ & 5284 \\
\hline 2875 вС & $62.2 \pm 2.7$ & $4203 \pm 21$ & 4824 & $3345 \mathrm{BC}$ & $75.8 \pm 2.7$ & $4557 \pm 20$ & 5294 \\
\hline 2885 BC & $58.7 \pm 3.8$ & $4239 \pm 29$ & 4834 & 3355 BC & $72.6 \pm 4.2$ & $4591 \pm 32$ & 5304 \\
\hline 2895 BC & $51.9 \pm 4.0$ & $4301 \pm 30$ & 4844 & $3365 \mathrm{BC}$ & $69.3 \pm 4.1$ & $4625 \pm 31$ & 5314 \\
\hline 2905 BC & $51.2 \pm 4.0$ & $4315 \pm 30$ & 4854 & $3375 \mathrm{BC}$ & $66.0 \pm 4.1$ & $4660 \pm 31$ & 5324 \\
\hline 2915 BC & $50.9 \pm 3.8$ & $4328 \pm 29$ & 4864 & $3385 \mathrm{BC}$ & $61.5 \pm 4.1$ & $4704 \pm 31$ & 5334 \\
\hline 2925 BC & $44.7 \pm 4.0$ & $4385 \pm 30$ & 4874 & $3395 \mathrm{BC}$ & $58.7 \pm 3.1$ & $4735 \pm 23$ & 5344 \\
\hline 2935 вС & $44.5 \pm 4.4$ & $4396 \pm 34$ & 4884 & $3405 \mathrm{BC}$ & $64.1 \pm 4.1$ & $4703 \pm 31$ & 5354 \\
\hline 2945 BC & $42.7 \pm 3.8$ & $4420 \pm 29$ & 4894 & $3415 \mathrm{BC}$ & $62.2 \pm 4.5$ & $4728 \pm 34$ & 5364 \\
\hline $2955 \mathrm{BC}$ & $50.0 \pm 2.7$ & $4373 \pm 21$ & 4904 & $3425 \mathrm{BC}$ & $68.5 \pm 3.4$ & $4690 \pm 26$ & 5374 \\
\hline 2965 BC & $49.6 \pm 3.8$ & $4386 \pm 29$ & 4914 & $3435 \mathrm{BC}$ & $70.6 \pm 2.9$ & $4684 \pm 22$ & 5384 \\
\hline $2975 \mathrm{BC}$ & $48.1 \pm 4.0$ & $4407 \pm 30$ & 4924 & $3445 \mathrm{BC}$ & $70.7 \pm 4.9$ & $4693 \pm 37$ & 5394 \\
\hline 2985 BC & $54.2 \pm 2.7$ & $4370 \pm 21$ & 4934 & $3455 \mathrm{BC}$ & $70.6 \pm 4.5$ & $4703 \pm 34$ & 5404 \\
\hline 2995 BC & $56.5 \pm 4.0$ & $4363 \pm 31$ & 4944 & $3465 \mathrm{BC}$ & $77.2 \pm 3.0$ & $4664 \pm 22$ & 5414 \\
\hline $3005 \mathrm{BC}$ & $56.3 \pm 4.0$ & $4374 \pm 31$ & 4954 & $3475 \mathrm{BC}$ & $84.3 \pm 2.9$ & $4621 \pm 21$ & 5424 \\
\hline 3015 BC & $54.9 \pm 3.9$ & $4395 \pm 30$ & 4964 & $3485 \mathrm{BC}$ & $84.4 \pm 2.9$ & $4629 \pm 22$ & 5434 \\
\hline $3025 \mathrm{BC}$ & $56.7 \pm 3.9$ & $4391 \pm 30$ & 4974 & $3495 \mathrm{BC}$ & $82.9 \pm 3.0$ & $4650 \pm 23$ & 5444 \\
\hline 3035 BC & $55.9 \pm 4.1$ & $4406 \pm 31$ & 4984 & $3505 \mathrm{BC}$ & $76.4 \pm 3.0$ & $4708 \pm 23$ & 5454 \\
\hline 3045 BC & $53.3 \pm 2.6$ & $4435 \pm 20$ & 4994 & 3515 BC & $73.5 \pm 2.9$ & $4740 \pm 22$ & 5464 \\
\hline $3055 \mathrm{BC}$ & $52.2 \pm 2.8$ & $4454 \pm 21$ & 5004 & $3525 \mathrm{BC}$ & $73.8 \pm 3.0$ & $4748 \pm 22$ & 5474 \\
\hline 3065 BC & $53.9 \pm 4.0$ & $4451 \pm 31$ & 5014 & $3535 \mathrm{BC}$ & $75.3 \pm 3.0$ & $4746 \pm 22$ & 5484 \\
\hline 3075 BC & $61.0 \pm 2.1$ & $4407 \pm 16$ & 5024 & $3545 \mathrm{BC}$ & $70.0 \pm 3.0$ & $4795 \pm 22$ & 5494 \\
\hline 3085 BC & $55.5 \pm 4.1$ & $4458 \pm 31$ & 5034 & $3555 \mathrm{BC}$ & $71.5 \pm 3.1$ & $4794 \pm 23$ & 5504 \\
\hline 3095 BC & $58.7 \pm 4.1$ & $4443 \pm 31$ & 5044 & 3565 вС & $76.8 \pm 3.0$ & $4764 \pm 22$ & 5514 \\
\hline 3105 BC & $49.0 \pm 4.1$ & $4527 \pm 31$ & 5054 & $3575 \mathrm{BC}$ & $76.4 \pm 3.0$ & $4777 \pm 22$ & 5524 \\
\hline $3115 \mathrm{BC}$ & $54.1 \pm 2.9$ & $4498 \pm 22$ & 5064 & $3585 \mathrm{BC}$ & $79.7 \pm 3.0$ & $4761 \pm 22$ & 5534 \\
\hline 3125 BC & $51.1 \pm 4.0$ & $4531 \pm 30$ & 5074 & $3595 \mathrm{BC}$ & $82.5 \pm 2.4$ & $4751 \pm 18$ & 5544 \\
\hline $3135 \mathrm{BC}$ & $52.5 \pm 3.9$ & $4529 \pm 29$ & 5084 & $3605 \mathrm{BC}$ & $85.7 \pm 2.6$ & $4737 \pm 20$ & 5554 \\
\hline 3145 BC & $49.3 \pm 4.0$ & $4563 \pm 30$ & 5094 & $3615 \mathrm{BC}$ & $90.6 \pm 2.3$ & $4710 \pm 17$ & 5564 \\
\hline $3155 \mathrm{BC}$ & $56.8 \pm 4.0$ & $4516 \pm 31$ & 5104 & $3625 \mathrm{BC}$ & $85.9 \pm 4.3$ & $4754 \pm 32$ & 5574 \\
\hline
\end{tabular}


TABLE 1. (Continued)

\begin{tabular}{|c|c|c|c|c|c|c|c|}
\hline Cal AD/BC & $\Delta^{14} \mathrm{C} \% 0$ & $\begin{array}{c}{ }^{14} \mathrm{C} \\
\text { age (BP) }\end{array}$ & Cal BP & $\mathrm{Cal} \mathrm{AD} / \mathrm{BC}$ & $\Delta{ }^{14} \mathrm{C} \% 0$ & $\begin{array}{l}{ }^{14} \mathrm{C} \\
\text { age (BP) }\end{array}$ & Cal BP \\
\hline $3635 \mathrm{BC}$ & $80.9 \pm 2.9$ & $4801 \pm 22$ & 5584 & $4105 \mathrm{BC}$ & $78.3 \pm 4.2$ & $5278 \pm 31$ & 6054 \\
\hline $3645 \mathrm{BC}$ & $75.8 \pm 3.0$ & $4849 \pm 22$ & 5594 & $4115 \mathrm{BC}$ & $69.4 \pm 2.9$ & $5353 \pm 22$ & 6064 \\
\hline $3655 \mathrm{BC}$ & $71.7 \pm 2.9$ & $4890 \pm 22$ & 5604 & $4125 \mathrm{BC}$ & $80.1 \pm 4.3$ & $5284 \pm 32$ & 6074 \\
\hline 3665 BC & $73.8 \pm 2.9$ & $4884 \pm 22$ & 5614 & $4135 \mathrm{BC}$ & $81.9 \pm 3.0$ & $5280 \pm 23$ & 6084 \\
\hline $3675 \mathrm{BC}$ & $68.5 \pm 2.4$ & $4933 \pm 18$ & 5624 & $4155 \mathrm{BC}$ & $85.4 \pm 4.3$ & $5273 \pm 32$ & 6104 \\
\hline $3685 \mathrm{BC}$ & $73.4 \pm 2.9$ & $4906 \pm 21$ & 5634 & $4165 \mathrm{BC}$ & $80.0 \pm 4.2$ & $5323 \pm 31$ & 6114 \\
\hline $3695 \mathrm{BC}$ & $75.8 \pm 2.9$ & $4897 \pm 22$ & 5644 & $4175 \mathrm{BC}$ & $75.2 \pm 4.4$ & $5369 \pm 33$ & 6124 \\
\hline $3705 \mathrm{BC}$ & $73.1 \pm 2.9$ & $4928 \pm 22$ & 5654 & $4185 \mathrm{BC}$ & $81.2 \pm 4.3$ & $5333 \pm 32$ & 6134 \\
\hline 3715 BC & $69.9 \pm 2.9$ & $4961 \pm 22$ & 5664 & 4195 BC & $84.7 \pm 3.0$ & $5318 \pm 22$ & 6144 \\
\hline $3725 \mathrm{BC}$ & $68.6 \pm 4.6$ & $4980 \pm 35$ & 5674 & $4205 \mathrm{BC}$ & $92.5 \pm 4.1$ & $5270 \pm 30$ & 6154 \\
\hline $3735 \mathrm{BC}$ & $74.4 \pm 3.0$ & $4947 \pm 23$ & 5684 & $4215 \mathrm{BC}$ & $93.9 \pm 4.4$ & $5269 \pm 33$ & 6164 \\
\hline $3745 \mathrm{BC}$ & $73.8 \pm 2.6$ & $4961 \pm 20$ & 5694 & $4225 \mathrm{BC}$ & $81.5 \pm 3.0$ & $5370 \pm 23$ & 6174 \\
\hline $3755 \mathrm{BC}$ & $76.2 \pm 2.9$ & $4953 \pm 22$ & 5704 & $4235 \mathrm{BC}$ & \pm 3.0 & $5380 \pm 22$ & 6184 \\
\hline $3765 \mathrm{BC}$ & $71.4 \pm 2.9$ & $4998 \pm 22$ & 5714 & $4245 \mathrm{BC}$ & $86.4 \pm 3.0$ & $5353 \pm 22$ & 6194 \\
\hline $3775 \mathrm{BC}$ & $71.5 \pm 3.0$ & $5007 \pm 22$ & 5724 & $4255 \mathrm{BC}$ & $78.8 \pm 5.0$ & $5419 \pm 37$ & 6204 \\
\hline $3785 \mathrm{BC}$ & $74.0 \pm 2.4$ & $4999 \pm 18$ & 5734 & $4265 \mathrm{BC}$ & $74.8 \pm 3.2$ & $5460 \pm 24$ & 6214 \\
\hline $3795 \mathrm{BC}$ & $73.7 \pm 3.1$ & $5011 \pm 23$ & 5744 & $4275 \mathrm{BC}$ & $79.5 \pm 5.0$ & $5433 \pm 37$ & 6224 \\
\hline $3805 \mathrm{BC}$ & $65.3 \pm 2.8$ & $5083 \pm 21$ & 5754 & $4285 \mathrm{BC}$ & $81.2 \pm 3.0$ & $5432 \pm 23$ & 6234 \\
\hline $3815 \mathrm{BC}$ & $65.4 \pm 3.9$ & $5092 \pm 30$ & 5764 & $4295 \mathrm{BC}$ & $85.4 \pm 4.5$ & $5409 \pm 33$ & 6244 \\
\hline $3825 \mathrm{BC}$ & $71.3 \pm 3.9$ & $5058 \pm 29$ & 5774 & $4305 \mathrm{BC}$ & $91.5 \pm 4.3$ & $5374 \pm 32$ & 6254 \\
\hline $3835 \mathrm{BC}$ & $73.0 \pm 4.1$ & $5055 \pm 31$ & 5784 & $4315 \mathrm{BC}$ & $92.0 \pm 4.4$ & $5380 \pm 33$ & 6264 \\
\hline $3845 \mathrm{BC}$ & $71.3 \pm 4.1$ & $5077 \pm 31$ & 5794 & $4325 \mathrm{BC}$ & $86.6 \pm 4.4$ & $5430 \pm 33$ & 6274 \\
\hline $3855 \mathrm{BC}$ & $71.7 \pm 2.4$ & $5083 \pm 18$ & 5804 & $4335 \mathrm{BC}$ & $80.8 \pm 3.0$ & $5484 \pm 23$ & 6284 \\
\hline $3865 \mathrm{BC}$ & $78.0 \pm 2.1$ & $5047 \pm 16$ & 5814 & $4345 \mathrm{BC}$ & $78.2 \pm 3.1$ & $5512 \pm 23$ & 6294 \\
\hline 3875 BC & $72.2 \pm 2.2$ & $5099 \pm 17$ & 5824 & $4355 \mathrm{BC}$ & 4.3 & $5553 \pm 33$ & 6304 \\
\hline 3885 BC & $85.3 \pm 2.7$ & $5011 \pm 20$ & 5834 & $4365 \mathrm{BC}$ & $73.7 \pm 4.2$ & $5564 \pm 32$ & 6314 \\
\hline 3895 BC & $82.3 \pm 3.5$ & $5044 \pm 26$ & 5844 & $4375 \mathrm{BC}$ & $68.8 \pm 4.3$ & $5576 \pm 22$ & 6324 \\
\hline $3905 \mathrm{BC}$ & $82.5 \pm 2.9$ & $5052 \pm 21$ & 5854 & $4385 \mathrm{BC}$ & $78.6 \pm 4.5$ & $5547 \pm 33$ & 6334 \\
\hline $3915 \mathrm{BC}$ & $81.9 \pm 2.8$ & $5066 \pm 21$ & 5864 & $4395 \mathrm{BC}$ & $75.3 \pm 4.4$ & $5582 \pm 33$ & 6344 \\
\hline $3925 \mathrm{BC}$ & $84.4 \pm 3.1$ & $5060 \pm 23$ & 5874 & $4405 \mathrm{BC}$ & $77.5 \pm 4.4$ & $5574 \pm 33$ & 6354 \\
\hline 3935 BC & $84.5 \pm 4.2$ & $5066 \pm 31$ & 5884 & 4415 BC & $73.2 \pm 4.4$ & $5616 \pm 33$ & 6364 \\
\hline 3945 BC & $79.9 \pm 2.4$ & $5113 \pm 18$ & 5894 & $4425 \mathrm{BC}$ & $=4.6$ & $5542 \pm 35$ & 6374 \\
\hline 3955 вC & $80.9 \pm 5.2$ & $5113 \pm 39$ & 5904 & $4435 \mathrm{BC}$ & 87.8 & $5527 \pm 34$ & 63 \\
\hline 3965 BC & $76.3 \pm 1.8$ & $5159 \pm 13$ & 5914 & 4445 BC & $83.5 \pm 4.4$ & $5569 \pm 32$ & 6394 \\
\hline 3975 BC & $72.9 \pm 2.6$ & $5192 \pm 19$ & 5924 & $4455 \mathrm{BC}$ & $79.6 \pm 4.6$ & $5608 \pm 34$ & 6404 \\
\hline $3985 \mathrm{BC}$ & $70.1 \pm 2.7$ & $5222 \pm 20$ & 5934 & $4465 \mathrm{BC}$ & $71.4 \pm 3.0$ & $5679 \pm 22$ & 6414 \\
\hline 3995 BC & $69.3 \pm 3.0$ & $5238 \pm 22$ & 5944 & $4475 \mathrm{BC}$ & $73.3 \pm 4.4$ & $5675 \pm 33$ & 6424 \\
\hline $4005 \mathrm{BC}$ & \pm 3.0 & $5251 \pm 23$ & 5954 & 4485 BC & $76.1 \pm 4.6$ & $5663 \pm 35$ & 6434 \\
\hline 4015 BC & $71.9 \pm 4.1$ & $5237 \pm 31$ & 59 & $4495 \mathrm{BC}$ & $75.4 \pm 4.4$ & $5678 \pm 33$ & 6444 \\
\hline $4025 \mathrm{BC}$ & $77.3 \pm 3.2$ & $5207 \pm 24$ & 5974 & $4505 \mathrm{BC}$ & 76. & 5681 & 64 \\
\hline 4035 BC & $76.0 \pm 2.7$ & $5226 \pm 20$ & 5984 & $4515 \mathrm{BC}$ & $77.2 \pm 4.4$ & $5684 \pm 33$ & 6464 \\
\hline $4045 \mathrm{BC}$ & $72.0 \pm 4.2$ & $5267 \pm 31$ & 5994 & $4525 \mathrm{BC}$ & $78.6 \pm 4.7$ & $5684 \pm 35$ & 6474 \\
\hline $4055 \mathrm{BC}$ & $80.7 \pm 3.7$ & $5211 \pm 28$ & 6004 & $4535 \mathrm{BC}$ & $79.0 \pm 4.2$ & $5690 \pm 32$ & 6484 \\
\hline $4065 \mathrm{BC}$ & $85.3 \pm 3.0$ & $5187 \pm 22$ & 6014 & $4545 \mathrm{BC}$ & $72.5 \pm 4.7$ & $5748 \pm 35$ & 6494 \\
\hline $4075 \mathrm{BC}$ & $79.0 \pm 4.2$ & $5243 \pm 31$ & 6024 & $4555 \mathrm{BC}$ & $72.8 \pm 4.4$ & $5756 \pm 33$ & 6504 \\
\hline $4085 \mathrm{BC}$ & $73.0 \pm 3.5$ & $5298 \pm 26$ & 6034 & $4565 \mathrm{BC}$ & $72.6 \pm 3.2$ & $5767 \pm 24$ & 6514 \\
\hline $4095 \mathrm{BC}$ & $74.5 \pm 3.0$ & $5296 \pm 22$ & 6044 & $4575 \mathrm{BC}$ & $75.9 \pm 3.0$ & $5752 \pm 23$ & 6524 \\
\hline
\end{tabular}


TABLE 1. (Continued)

\begin{tabular}{|c|c|c|c|c|c|c|c|}
\hline \multicolumn{4}{|c|}{${ }^{14} \mathrm{C}$} & \multicolumn{4}{|c|}{${ }^{14} \mathrm{C}$} \\
\hline Cal $A D / B C$ & $\Delta^{14} \mathrm{C} \%$ o & age (BP) & Cal BP & Cal $\mathrm{AD} / \mathrm{BC}$ & $\Delta^{14} \mathrm{C} \% 0$ & age (BP) & Cal BP \\
\hline $4585 \mathrm{BC}$ & $77.1 \pm 3.1$ & $5753 \pm 23$ & 6534 & $5055 \mathrm{BC}$ & $89.3 \pm 4.1$ & $6119 \pm 33$ & 7004 \\
\hline 4595 вс & $76.4 \pm 4.4$ & $5768 \pm 33$ & 6544 & $5065 \mathrm{BC}$ & $83.4 \pm 4.0$ & $6172 \pm 32$ & 7014 \\
\hline $4605 \mathrm{BC}$ & $77.9 \pm 4.3$ & $5767 \pm 32$ & 6554 & $5075 \mathrm{BC}$ & $82.4 \pm 4.2$ & $6189 \pm 34$ & 7024 \\
\hline $4615 \mathrm{BC}$ & $79.6 \pm 4.7$ & $5764 \pm 35$ & 6564 & $5085 \mathrm{BC}$ & $80.3 \pm 4.1$ & $6215 \pm 33$ & 7034 \\
\hline 4625 вC & $74.7 \pm 2.7$ & $5810 \pm 21$ & 6574 & $5095 \mathrm{BC}$ & $86.9 \pm 2.9$ & $6176 \pm 24$ & 7044 \\
\hline $4635 \mathrm{BC}$ & $78.9 \pm 3.7$ & $5788 \pm 28$ & 6584 & $5105 \mathrm{BC}$ & $82.9 \pm 4.1$ & $6215 \pm 33$ & 7054 \\
\hline $4645 \mathrm{BC}$ & $81.9 \pm 2.3$ & $5775 \pm 17$ & 6594 & $5115 \mathrm{BC}$ & $92.6 \pm 4.0$ & $6153 \pm 32$ & 7064 \\
\hline $4655 \mathrm{BC}$ & $82.1 \pm 3.1$ & $5784 \pm 23$ & 6604 & $5125 \mathrm{BC}$ & $85.0 \pm 4.0$ & $6219 \pm 32$ & 7074 \\
\hline $4665 \mathrm{BC}$ & $87.4 \pm 4.4$ & $5754 \pm 32$ & 6614 & $5135 \mathrm{BC}$ & $94.8 \pm 3.9$ & $6157 \pm 31$ & 7084 \\
\hline $4675 \mathrm{BC}$ & $87.3 \pm 4.8$ & $5764 \pm 35$ & 6624 & $5145 \mathrm{BC}$ & $95.6 \pm 3.0$ & $6160 \pm 24$ & 7094 \\
\hline $4685 \mathrm{BC}$ & $86.2 \pm 2.6$ & $5782 \pm 19$ & 6634 & $5165 \mathrm{BC}$ & $92.6 \pm 4.1$ & $6202 \pm 33$ & 7114 \\
\hline $4695 \mathrm{BC}$ & $80.1 \pm 3.2$ & $5837 \pm 24$ & 6644 & $5175 \mathrm{BC}$ & $99.0 \pm 4.0$ & $6164 \pm 32$ & 7124 \\
\hline $4705 \mathrm{BC}$ & $83.6 \pm 3.0$ & $5821 \pm 23$ & 6654 & $5185 \mathrm{BC}$ & $106.7 \pm 2.9$ & $6118 \pm 24$ & 7134 \\
\hline $4715 \mathrm{BC}$ & $79.8 \pm 4.3$ & $5859 \pm 32$ & 6664 & $5195 \mathrm{BC}$ & $106.7 \pm 4.0$ & $6128 \pm 33$ & 7144 \\
\hline $4725 \mathrm{BC}$ & $78.4 \pm 4.4$ & $5879 \pm 33$ & 6674 & $5205 \mathrm{BC}$ & $108.9 \pm 4.3$ & $6122 \pm 34$ & 7154 \\
\hline $4735 \mathrm{BC}$ & $80.0 \pm 4.3$ & $5877 \pm 32$ & 6684 & $5215 \mathrm{BC}$ & $94.6 \pm 4.2$ & $6236 \pm 34$ & 7164 \\
\hline $4745 \mathrm{BC}$ & $87.1 \pm 4.4$ & $5834 \pm 33$ & 6694 & $5225 \mathrm{BC}$ & $89.7 \pm 4.3$ & $6281 \pm 35$ & 7174 \\
\hline $4755 \mathrm{BC}$ & $88.7 \pm 4.4$ & $5832 \pm 32$ & 6704 & $5235 \mathrm{BC}$ & $98.1 \pm 4.2$ & $6230 \pm 34$ & 7184 \\
\hline $4765 \mathrm{BC}$ & $81.6 \pm 4.3$ & $5895 \pm 32$ & 6714 & $5245 \mathrm{BC}$ & $96.0 \pm 2.9$ & $6255 \pm 24$ & 7194 \\
\hline $4775 \mathrm{BC}$ & $83.7 \pm 4.4$ & $5889 \pm 33$ & 6724 & $5255 \mathrm{BC}$ & $97.1 \pm 2.9$ & $6256 \pm 23$ & 7204 \\
\hline $4785 \mathrm{BC}$ & $84.3 \pm 4.3$ & $5894 \pm 32$ & 6734 & $5265 \mathrm{BC}$ & $92.4 \pm 4.0$ & $6300 \pm 32$ & 7214 \\
\hline $4795 \mathrm{BC}$ & $82.8 \pm 4.3$ & $5914 \pm 32$ & 6744 & $5275 \mathrm{BC}$ & $90.9 \pm 2.7$ & $6321 \pm 22$ & 7224 \\
\hline 4805 вС & $80.9 \pm 4.6$ & $5939 \pm 34$ & 6754 & $5285 \mathrm{BC}$ & $83.0 \pm 2.8$ & $6389 \pm 23$ & 7234 \\
\hline $4815 \mathrm{BC}$ & $83.8 \pm 4.5$ & $5926 \pm 34$ & 6764 & $5295 \mathrm{BC}$ & $89.3 \pm 4.2$ & $6352 \pm 34$ & 7244 \\
\hline $4825 \mathrm{BC}$ & $80.5 \pm 4.4$ & $5961 \pm 33$ & 6774 & $5305 \mathrm{BC}$ & $86.7 \pm 4.3$ & $6381 \pm 35$ & 7254 \\
\hline $4835 \mathrm{BC}$ & $82.6 \pm 4.3$ & $5955 \pm 32$ & 6784 & $5315 \mathrm{BC}$ & $90.7 \pm 3.0$ & $6361 \pm 24$ & 7264 \\
\hline $4845 \mathrm{BC}$ & $81.3 \pm 4.6$ & $5974 \pm 34$ & 6794 & $5325 \mathrm{BC}$ & $89.3 \pm 3.0$ & $6381 \pm 24$ & 7274 \\
\hline $4855 \mathrm{BC}$ & $78.8 \pm 3.1$ & $6003 \pm 23$ & 6804 & $5335 \mathrm{BC}$ & $85.1 \pm 4.0$ & $6422 \pm 32$ & 7284 \\
\hline 4865 вс & $81.3 \pm 4.4$ & $5994 \pm 33$ & 6814 & $5345 \mathrm{BC}$ & $85.7 \pm 3.0$ & $6428 \pm 24$ & 7294 \\
\hline $4875 \mathrm{BC}$ & $77.8 \pm 4.5$ & $6029 \pm 33$ & 6824 & $5355 \mathrm{BC}$ & $82.4 \pm 3.0$ & $6462 \pm 24$ & 7304 \\
\hline 4885 вC & $93.1 \pm 4.6$ & $5926 \pm 34$ & 6834 & $5365 \mathrm{BC}$ & $91.0 \pm 4.2$ & $6408 \pm 34$ & 7314 \\
\hline 4895 вС & $86.4 \pm 3.3$ & $5985 \pm 25$ & 6844 & $5375 \mathrm{BC}$ & $94.7 \pm 4.4$ & $6390 \pm 36$ & 7324 \\
\hline $4905 \mathrm{BC}$ & $88.1 \pm 2.6$ & $5982 \pm 19$ & 6854 & $5385 \mathrm{BC}$ & $93.1 \pm 4.9$ & $6412 \pm 39$ & 7334 \\
\hline 4915 BC & $85.0 \pm 3.5$ & $6015 \pm 26$ & 6864 & $5395 \mathrm{BC}$ & $96.0 \pm 4.2$ & $6400 \pm 34$ & 7344 \\
\hline $4925 \mathrm{BC}$ & $83.8 \pm 4.5$ & $6033 \pm 33$ & 6874 & $5405 \mathrm{BC}$ & $95.2 \pm 4.2$ & $6416 \pm 34$ & 7354 \\
\hline $4935 \mathrm{BC}$ & $89.8 \pm 3.2$ & $5999 \pm 24$ & 6884 & $5415 \mathrm{BC}$ & $100.9 \pm 3.0$ & $6384 \pm 24$ & 7364 \\
\hline $4945 \mathrm{BC}$ & $84.4 \pm 2.3$ & $6047 \pm 17$ & 6894 & $5425 \mathrm{BC}$ & $99.8 \pm 3.1$ & $6402 \pm 25$ & 7374 \\
\hline 4955 вС & $86.4 \pm 3.1$ & $6042 \pm 23$ & 6904 & $5435 \mathrm{BC}$ & $89.7 \pm 4.1$ & $6485 \pm 33$ & 7384 \\
\hline $4965 \mathrm{BC}$ & $84.8 \pm 3.3$ & $6065 \pm 24$ & 6914 & $5445 \mathrm{BC}$ & $79.8 \pm 3.1$ & $6568 \pm 25$ & 7394 \\
\hline $4975 \mathrm{BC}$ & $87.8 \pm 3.1$ & $6053 \pm 23$ & 6924 & $5455 \mathrm{BC}$ & $81.7 \pm 4.3$ & $6564 \pm 34$ & 7404 \\
\hline $4985 \mathrm{BC}$ & $88.3 \pm 3.3$ & $6058 \pm 25$ & 6934 & $5465 \mathrm{BC}$ & $87.1 \pm 3.0$ & $6534 \pm 24$ & 7414 \\
\hline 4995 BC & $85.1 \pm 4.3$ & $6092 \pm 32$ & 6944 & $5475 \mathrm{BC}$ & $83.7 \pm 4.3$ & $6568 \pm 35$ & 7424 \\
\hline $5005 \mathrm{BC}$ & $83.3 \pm 4.1$ & $6115 \pm 33$ & 6954 & 5485 вС & $86.9 \pm 4.4$ & $6554 \pm 35$ & 7434 \\
\hline 5015 вС & $88.4 \pm 4.2$ & $6087 \pm 34$ & 6964 & $5495 \mathrm{BC}$ & $83.0 \pm 4.3$ & $6593 \pm 35$ & 7444 \\
\hline $5025 \mathrm{BC}$ & $85.6 \pm 4.1$ & $6117 \pm 33$ & 6974 & $5505 \mathrm{BC}$ & $81.8 \pm 4.3$ & $6612 \pm 35$ & 7454 \\
\hline 5035 вс & $87.2 \pm 4.3$ & $6115 \pm 34$ & 6984 & $5515 \mathrm{BC}$ & $87.0 \pm 4.3$ & $6583 \pm 35$ & 7464 \\
\hline $5045 \mathrm{BC}$ & $85.4 \pm 4.3$ & $6138 \pm 34$ & 6994 & $5525 \mathrm{BC}$ & $75.5 \pm 4.2$ & $6678 \pm 34$ & 7474 \\
\hline
\end{tabular}


TABLE 1. (Continued)

\begin{tabular}{lccl|llll}
\hline & \multicolumn{3}{c|}{${ }^{14} \mathrm{C}$} & & & \\
Cal AD/BC & $\Delta^{14} \mathrm{C} \% 0$ & age (BP) & Cal BP & Cal AD/BC & $\Delta^{14} \mathrm{C} \% 0$ & age (BP) & Cal BP \\
\hline $5535 \mathrm{BC}$ & $79.4 \pm 4.4$ & $6659 \pm 35$ & 7484 & $5775 \mathrm{BC}$ & $69.2 \pm 2.9$ & $6969 \pm 22$ & 7724 \\
$5545 \mathrm{BC}$ & $77.9 \pm 4.4$ & $6680 \pm 35$ & 7494 & $5785 \mathrm{BC}$ & $72.8 \pm 2.2$ & $6953 \pm 17$ & 7734 \\
$5555 \mathrm{BC}$ & $89.8 \pm 3.3$ & $6604 \pm 25$ & 7504 & $5795 \mathrm{BC}$ & $75.1 \pm 2.4$ & $6947 \pm 18$ & 7744 \\
$5565 \mathrm{BC}$ & $90.7 \pm 3.3$ & $6606 \pm 25$ & 7514 & $5805 \mathrm{BC}$ & $71.3 \pm 2.4$ & $6982 \pm 19$ & 7754 \\
$5575 \mathrm{BC}$ & $83.5 \pm 2.4$ & $6670 \pm 18$ & 7524 & $5815 \mathrm{BC}$ & $69.9 \pm 2.1$ & $7004 \pm 17$ & 7764 \\
$5585 \mathrm{BC}$ & $77.7 \pm 3.3$ & $6721 \pm 25$ & 7534 & $5825 \mathrm{BC}$ & $75.8 \pm 2.5$ & $6969 \pm 20$ & 7774 \\
$5595 \mathrm{BC}$ & $76.1 \pm 3.4$ & $6742 \pm 26$ & 7544 & $5835 \mathrm{BC}$ & $75.2 \pm 2.6$ & $6983 \pm 20$ & 7784 \\
$5605 \mathrm{BC}$ & $70.8 \pm 2.5$ & $6793 \pm 19$ & 7554 & $5845 \mathrm{BC}$ & $76.8 \pm 2.6$ & $6981 \pm 21$ & 7794 \\
$5615 \mathrm{BC}$ & $74.9 \pm 2.2$ & $6774 \pm 17$ & 7564 & $5855 \mathrm{BC}$ & $70.7 \pm 4.7$ & $7035 \pm 38$ & 7804 \\
$5625 \mathrm{BC}$ & $74.9 \pm 2.7$ & $6780 \pm 21$ & 7574 & $5865 \mathrm{BC}$ & $76.0 \pm 3.9$ & $7005 \pm 31$ & 7814 \\
$5635 \mathrm{BC}$ & $70.8 \pm 2.4$ & $6822 \pm 19$ & 7584 & $5875 \mathrm{BC}$ & $76.3 \pm 4.2$ & $7012 \pm 34$ & 7824 \\
$5645 \mathrm{BC}$ & $75.2 \pm 2.6$ & $6799 \pm 20$ & 7594 & $5885 \mathrm{BC}$ & $68.9 \pm 4.2$ & $7077 \pm 34$ & 7834 \\
$5655 \mathrm{BC}$ & $76.4 \pm 2.8$ & $6800 \pm 21$ & 7604 & $5895 \mathrm{BC}$ & $68.0 \pm 4.6$ & $7094 \pm 37$ & 7844 \\
$5665 \mathrm{BC}$ & $75.8 \pm 2.1$ & $6815 \pm 16$ & 7614 & $5905 \mathrm{BC}$ & $74.8 \pm 3.1$ & $7053 \pm 25$ & 7854 \\
$5675 \mathrm{BC}$ & $75.2 \pm 2.7$ & $6828 \pm 20$ & 7624 & $5915 \mathrm{BC}$ & $85.0 \pm 3.2$ & $6986 \pm 26$ & 7864 \\
$5685 \mathrm{BC}$ & $71.7 \pm 3.1$ & $6863 \pm 24$ & 7634 & $5925 \mathrm{BC}$ & $87.0 \pm 3.0$ & $6981 \pm 24$ & 7874 \\
$5695 \mathrm{BC}$ & $71.2 \pm 2.7$ & $6875 \pm 21$ & 7644 & $5935 \mathrm{BC}$ & $81.5 \pm 5.2$ & $7032 \pm 42$ & 7884 \\
$5705 \mathrm{BC}$ & $70.9 \pm 2.5$ & $6885 \pm 20$ & 7654 & $5945 \mathrm{BC}$ & $75.3 \pm 4.2$ & $7088 \pm 34$ & 7894 \\
$5715 \mathrm{BC}$ & $69.5 \pm 3.3$ & $6907 \pm 26$ & 7664 & $5955 \mathrm{BC}$ & $75.3 \pm 4.1$ & $7097 \pm 33$ & 7904 \\
$5725 \mathrm{BC}$ & $73.7 \pm 3.1$ & $6884 \pm 24$ & 7674 & $5965 \mathrm{BC}$ & $73.9 \pm 4.4$ & $7118 \pm 35$ & 7914 \\
$5735 \mathrm{BC}$ & $69.4 \pm 2.5$ & $6929 \pm 19$ & 7684 & $5975 \mathrm{BC}$ & $70.2 \pm 5.0$ & $7155 \pm 40$ & 7924 \\
$5745 \mathrm{BC}$ & $70.2 \pm 2.3$ & $6933 \pm 18$ & 7694 & $5985 \mathrm{BC}$ & $67.8 \pm 2.8$ & $7182 \pm 23$ & 7934 \\
$5755 \mathrm{BC}$ & $70.8 \pm 2.6$ & $6938 \pm 20$ & 7704 & $5995 \mathrm{BC}$ & $65.2 \pm 4.8$ & $7212 \pm 36$ & 7944 \\
$5765 \mathrm{BC}$ & $73.1 \pm 2.2$ & $6931 \pm 17$ & 7714 & $6000 \mathrm{BC}$ & $75.6 \pm 3.3$ & $7141 \pm 25$ & 7949 \\
& & & & & & &
\end{tabular}

